\title{
On simultaneous inhomogeneous Diophantine approximation
}

\author{
by \\ Yann Bugeaud (Strasbourg) and Nicolas Chevallier (Mulhouse)
}

1. Introduction. Let $c$ and $\tau$ be real numbers. A pair $(\alpha, \xi)$ of real numbers is called $(c, \tau)$-approximable if there exist infinitely many integers $q$ such that $\|q \alpha-\xi\|<c|q|^{-\tau}$. If $\alpha$ and $\xi$ are irrational real numbers such that $\xi$ is not of the form $\xi=m \alpha+n$ for integers $m, n$, then a theorem of Minkowski (cf. e.g. [5, p. 48]) asserts that there exist infinitely many integers $q$ such that

$$
\|q \alpha-\xi\|<\frac{1}{4|q|}
$$

where $\|\cdot\|$ denotes the distance to the nearest integer. Thus, almost all pairs $(\alpha, \xi)$ are $(1 / 4,1)$-approximable. Here and throughout the present paper, "almost all" refers to the Lebesgue measure on the ambient space.

Dodson [7] proved that the set of pairs $(\alpha, \xi)$ in $\mathbb{R}^{2}$ which are $(1, \tau)$ approximable for some $\tau>1$ has Lebesgue measure zero and Hausdorff dimension two. This is the so-called "doubly metric" statement, and we may as well adopt two further points of view, which are "singly metric". The first one consists in considering that $\xi$ is fixed and in looking at the set of real numbers $\alpha$ for which $(\alpha, \xi)$ is $(1, \tau)$-approximable. This is the most classical point of view in inhomogeneous Diophantine approximation, which has been considered by many authors. For instance, Levesley [13], extending a classical result of Jarník [10] and Besicovitch [3] dealing with the homogeneous case $\xi=0$, proved that, for any $\tau>1$ and any fixed $\xi$, the Hausdorff dimension of the set of $\alpha$ such that the pair $(\alpha, \xi)$ is $(1, \tau)$ approximable is equal to $2 /(1+\tau)$. We stress that this value does not depend on $\xi$.

The second point of view has been investigated by Bernik and Dodson $[2$, p. 105]. Their results have been improved upon by Schmeling and Troubetzkoy [14] and, independently and at the same time (by means of a

2000 Mathematics Subject Classification: 11J83, 11J20. 
different approach), by Bugeaud [4] who showed that, for any $\tau>1$ and any fixed irrational number $\alpha$, the set

$$
\mathcal{T}_{\tau}(\alpha):=\left\{\xi \in \mathbb{R}:\|n \alpha-\xi\|<1 / n^{\tau} \text { for infinitely many } n \in \mathbb{Z}_{\geq 1}\right\}
$$

has Hausdorff dimension $1 / \tau$. We stress that this value does not depend on $\alpha$.

These questions can as well be addressed in a multidimensional setting, by considering either inhomogeneous approximation of linear forms, or simultaneous rational inhomogeneous approximation, or even simultaneous inhomogeneous approximation of linear forms. In the "doubly metric" case and in the first "singly metric" case mentioned above, satisfactory answers have been given by Dodson [7] and Levesley [13], respectively. However, no multidimensional extension of the statements established in [14] and [4] has been studied up to now, and it is the purpose of the present work to report various results on this question.

Let $k \geq 1$ be an integer and let $\underline{\alpha}=\left(\alpha_{1}, \ldots, \alpha_{k}\right)$ be a $k$-tuple of real numbers. For real numbers $v>1$ and $w>0$, we set

$$
\begin{array}{r}
\mathcal{V}_{v}(\underline{\alpha}):=\left\{\xi \in \mathbb{R}:\left\|n_{1} \alpha_{1}+\cdots+n_{k} \alpha_{k}-\xi\right\|<\frac{c}{\left(\max _{1 \leq i \leq k}\left\{\left|n_{i}\right|\right\}\right)^{v}}\right. \\
\text { for some } \left.c>0 \text { and infinitely many }\left(n_{1}, \ldots, n_{k}\right) \in \mathbb{Z}^{k}\right\}
\end{array}
$$

and

$$
\begin{aligned}
& \mathcal{W}_{w}(\underline{\alpha}):=\left\{\left(\xi_{1}, \ldots, \xi_{k}\right) \in \mathbb{R}^{k}: \max _{1 \leq i \leq k}\left\{\left\|n \alpha_{i}-\xi_{i}\right\|\right\}<c /|n|^{w}\right. \\
&\text { for some } c>0 \text { and infinitely many } n \in \mathbb{Z}\} .
\end{aligned}
$$

Observe that, for $k=1$ and $w=v>1$, neither set coincides with $\mathcal{T}_{v}(\alpha)$. Actually, it is much more natural to work with $\mathcal{V}_{v}((\alpha))$ than with $\mathcal{T}_{v}(\alpha)$, since, for instance, the former set is clearly invariant under rational translations. In addition, there is no reason for considering only the positive integers. However, it is easily seen that both sets have the same Hausdorff dimension, namely $1 / v$.

First, we recall a result of Cassels, which describes the "almost everywhere" situation. According to [5, p. 92], a system $L_{j}(\underline{x})$ of $n$ linear forms in $m$ variables is singular if, for every $\varepsilon>0$, the set of inequalities

$$
\left\|L_{j}(\underline{x})\right\|<\varepsilon X^{-m / n}, \quad\left|x_{i}\right| \leq X,
$$

has a non-zero integer solution $\underline{x}$ for all $X$ sufficiently large (in terms of $\varepsilon$ ). Otherwise, the system is called regular (see Section 6 below). It follows from the Borel-Cantelli lemma (see e.g. [5, p. 92]) that the set of singular systems has Lebesgue measure zero in the $m n$-dimensional space. The following result follows from [5, Theorem XIII, p. 93], by taking $n=1$ or $m=1$. 
Theorem A. Let $k \geq 1$ be an integer. For almost all real $k$-tuples $\underline{\alpha}=$ $\left(\alpha_{1}, \ldots, \alpha_{k}\right)$, we have

$$
\mathcal{V}_{k}(\underline{\alpha})=\mathbb{R} \quad \text { and } \quad \mathcal{W}_{1 / k}(\underline{\alpha})=\mathbb{R}^{k} .
$$

In the present work, we are mainly interested in exceptional $k$-tuples, that is, $k$-tuples $\underline{\alpha}=\left(\alpha_{1}, \ldots, \alpha_{k}\right)$ for which either $\mathcal{V}_{k}(\underline{\alpha})$ is considerably smaller than $\mathbb{R}$, or $\mathcal{W}_{1 / k}(\underline{\alpha})$ is considerably smaller than $\mathbb{R}^{k}$. These are necessarily singular tuples, and since singular $k$-tuples only exist when $k \geq 2$ ([5, p. 94]), $k$ must be greater than or equal to 2 . We prove that, for $k=2$ or 3 , there exist real $k$-tuples $\underline{\alpha}=\left(\alpha_{1}, \ldots, \alpha_{k}\right)$ with $1, \alpha_{1}, \ldots, \alpha_{k}$ linearly independent over the rationals and such that the Hausdorff dimension of the set $\mathcal{V}_{k}(\underline{\alpha})$ is equal to $1 / k$. In view of the results from [14] and [4], the dimension cannot be smaller. Furthermore, we prove that, for any arbitrarily small positive $w$, there exist real $k$-tuples $\underline{\alpha}$ with $1, \alpha_{1}, \ldots, \alpha_{k}$ linearly independent over the rationals and such that the set $\mathcal{W}_{w}(\underline{\alpha})$ is small, in the sense that its Hausdorff dimension is at most equal to 1 . This considerably strengthens and generalizes a result of Khinchin, who proved [11] (see also [5, Theorem XV]) that, for $k=2$ and $w>0$ arbitrary, there exist pairs $\left(\alpha_{1}, \alpha_{2}\right)$ such that the set $\mathcal{W}_{w}\left(\alpha_{1}, \alpha_{2}\right)$ is not $\mathbb{R}^{2}$.

The present paper is organized as follows. Section 2 is devoted to the statement of the results, together with some additional remarks. Theorem 1 , concerning inhomogeneous approximation of linear forms, is proved in Section 3. Section 4 is devoted to the proof of Theorem 2, on inhomogeneous simultaneous rational approximation, and Section 5 to the proof of Theorem 3, which shows that, to some extent, Theorem 2 is best possible. Finally, Theorems 4 and 5, which deal with metric results, are proved in Section 6 .

2. Statement of the results. We begin by stating an application of the Hausdorff-Cantelli lemma, that provides us with upper bounds for the Hausdorff dimension by an easy covering argument.

Proposition 1. Let $k \geq 2$ be an integer and $\underline{\alpha}=\left(\alpha_{1}, \ldots, \alpha_{k}\right)$ be a real $k$-tuple. Let $v$ and $w$ be positive real numbers. Then

$$
\operatorname{dim} \mathcal{V}_{v}(\underline{\alpha}) \leq \min \{1, k / v\} \quad \text { and } \quad \operatorname{dim} \mathcal{W}_{w}(\underline{\alpha}) \leq \min \{k, 1 / w\} .
$$

Often, the upper bounds given by the Hausdorff-Cantelli lemma coincide with the exact value of the Hausdorff dimension, thus Proposition 1 (whose easy proof is postponed to the beginning of Section 6) gives the expected values for the Hausdorff dimensions of $\mathcal{V}_{v}(\underline{\alpha})$ and $\mathcal{W}_{w}(\underline{\alpha})$.

We first turn our attention to inhomogeneous approximation of linear forms. 
THEOREM 1. Let $k=2$ or 3 . Let $v>1$ be real. There exist real $k$-tuples $\underline{\alpha}=\left(\alpha_{1}, \ldots, \alpha_{k}\right)$ such that $1, \alpha_{1}, \ldots, \alpha_{k}$ are linearly independent over the rationals and

$$
\operatorname{dim} \mathcal{V}_{v}(\underline{\alpha})=1 / v \text {. }
$$

It follows from the result on the sets $\mathcal{T}_{\tau}(\alpha)$ recalled in the introduction that, for any real number $v>1$ and any irrational number $\alpha_{1}$, the Hausdorff dimension of $\mathcal{V}_{v}\left(\alpha_{1}\right)$ is $1 / v$. Consequently, the Hausdorff dimension of any set $\mathcal{V}_{v}\left(\alpha_{1}, \ldots, \alpha_{k}\right)$ as in Theorem 1 is at least $1 / v$.

The assumption that $1, \alpha_{1}, \ldots, \alpha_{k}$ are linearly independent over the rationals (which occurs in the statements of Theorems 1 to 3 ) ensures that the result is non-trivial, since e.g. $\operatorname{dim} \mathcal{V}_{v}(\alpha, 2 \alpha, \ldots, k \alpha)=1 / v$ for any $v>1$, any $k \geq 2$, and any irrational real number $\alpha$, by the results from [14] and [4].

Theorem 1 shows that there exist real $k$-tuples $\underline{\alpha}$ for which the upper bound given by Proposition 1 for the Hausdorff dimension of $\mathcal{V}_{v}(\underline{\alpha})$ is considerably larger than the exact value.

We are convinced that Theorem 1 holds for all integers $k \geq 2$. However, we only succeeded in establishing it for $k=2$ and $k=3$. Our method of proof is quite technical and it presumably works as well for $k \geq 4$.

REMARK. An interesting question remains. For any real numbers $v$ and $d$ with $v>1$ and $1 / v<d<k / v$, does there exist a $k$-tuple $\underline{\alpha}$ for which $\operatorname{dim} \mathcal{V}_{v}(\underline{\alpha})=d ?$

We now consider inhomogeneous simultaneous rational approximation, and we state a slightly sharper result than announced in the introduction. For any function $\phi: \mathbb{Z}_{\geq 1} \rightarrow \mathbb{R}_{>0}$ and any $k$-tuple of real numbers $\underline{\alpha}=$ $\left(\alpha_{1}, \ldots, \alpha_{k}\right)$, set

$$
\mathcal{W}_{\phi}(\underline{\alpha}):=\left\{\left(\xi_{1}, \ldots, \xi_{k}\right) \in \mathbb{R}^{k}: \max _{1 \leq i \leq k}\left\{\left\|n \alpha_{i}-\xi_{i}\right\|\right\}<\phi(|n|)\right.
$$

for infinitely many $n \in \mathbb{Z}\}$.

With this notation, for any positive real number $w$, the union of the sets $\mathcal{W}_{x \mapsto c x^{-w}}(\underline{\alpha})$ taken over the positive real numbers $c$ is simply $\mathcal{W}_{w}(\underline{\alpha})$.

TheOREM 2. Let $k \geq 2$ be an integer. Let $\phi: \mathbb{Z}_{\geq 1} \rightarrow \mathbb{R}_{>0}$ be a function tending to 0 at infinity. There exist real $k$-tuples $\underline{\alpha}=\left(\alpha_{1}, \ldots, \alpha_{k}\right)$ such that $1, \alpha_{1}, \ldots, \alpha_{k}$ are linearly independent over the rationals and

$$
\operatorname{dim} \mathcal{W}_{\phi}(\underline{\alpha}) \leq 1 \text {. }
$$

Consequently, there exist $k$-tuples $\underline{\alpha}$ for which

$$
\operatorname{dim} \mathcal{W}_{w}(\underline{\alpha}) \leq 1 \quad \text { for every } w>0 .
$$

We point out that, in Theorem 2, the function $\phi$ can tend to 0 arbitrarily slowly (in particular, $w$ can be taken arbitrarily close to 0 ), and that it is not assumed to be non-increasing. 
The existence of pairs $\left(\alpha_{1}, \alpha_{2}\right)$ of real numbers such that $\mathcal{W}_{\phi}\left(\alpha_{1}, \alpha_{2}\right)$ is not $\mathbb{R}^{2}$ is due to Khinchin [11]. It follows from the proof of Theorem XV from [5], combined with metric results of Schmidt on badly approximable pairs [15], that there exist pairs $\left(\alpha_{1}, \alpha_{2}\right)$ for which the complement of $\mathcal{W}_{\phi}\left(\alpha_{1}, \alpha_{2}\right)$ has Hausdorff dimension two (in $\mathbb{R}^{2}$ ). As far as we are aware, the existence of pairs $\left(\alpha_{1}, \alpha_{2}\right)$ such that $\mathcal{W}_{\phi}\left(\alpha_{1}, \alpha_{2}\right)$ has Lebesgue measure zero was not established up to now. Theorem 2 is even stronger.

We emphasize that the constructions given in the proofs of Theorems 1 and 2 are effective, thus, it is possible to give explicit examples of $k$-tuples satisfying the conclusions of these theorems. Obviously, such $k$-tuples are singular. They illustrate how the behaviour of singular systems can differ from the behaviour of regular systems. In the light of Theorem A, Theorems 1 and 2 may appear somehow surprising.

It turns out that the upper bound for the dimension obtained in Theorem 2 is sharp.

TheOREM 3. Let $k \geq 2$ be an integer. For any real number $w>0$ and any real $k$-tuple $\underline{\alpha}=\left(\alpha_{1}, \ldots, \alpha_{k}\right)$ with $1, \alpha_{1}, \ldots, \alpha_{k}$ linearly independent over the rationals, we have

$$
\begin{array}{ll}
\operatorname{dim} \mathcal{W}_{w}(\underline{\alpha})=1 / w & \text { if } w \geq 1, \\
\operatorname{dim} \mathcal{W}_{w}(\underline{\alpha}) \geq 1 & \text { if } 0<w \leq 1 .
\end{array}
$$

Actually, we prove a slightly sharper result than Theorem 3. The proof of Theorem 3 follows from the application of the (easy half of) Frostman's lemma to a suitable Cantor-type set, constructed inductively and contained in $\mathcal{W}_{w}(\underline{\alpha})$. This can be viewed as a (difficult) extension of the proof of the main result from [4].

Unlike the case of linear forms, the Hausdorff dimension of $\mathcal{W}_{w}(\underline{\alpha})$ does not depend on the $k$-tuple $\underline{\alpha}$, provided that $w$ is large enough, namely greater than or equal to 1.

We complement Theorems 1 to 3 by two statements valid for almost all $k$-tuples.

TheORem 4. Let $k \geq 2$ be an integer and $v \geq k$ be a real number. For almost every real $k$-tuple $\underline{\alpha}=\left(\alpha_{1}, \ldots, \alpha_{k}\right)$, we have

$$
\operatorname{dim} \mathcal{V}_{v}(\underline{\alpha})=k / v \text {. }
$$

In view of Theorem 3 , Theorem 5 below is interesting only in the range $1 / k<w<1$.

THeOREm 5. Let $k \geq 2$ be an integer and $w \geq 1 / k$ be a real number. For almost every real $k$-tuple $\underline{\alpha}=\left(\alpha_{1}, \ldots, \alpha_{k}\right)$, we have

$$
\operatorname{dim} \mathcal{W}_{w}(\underline{\alpha})=1 / w
$$


Theorems 4 and 5 are particular cases of a more general statement on systems of linear forms (see Theorem 6 in Section 6). They show that the upper bounds given by Proposition 1 are "almost always" the exact values of the dimension.

Theorems 1 and 2 state that, for $k \geq 2, v>1$ and $w<1$, the Hausdorff dimensions of the sets $\mathcal{V}_{v}(\underline{\alpha})$ and $\mathcal{W}_{w}(\underline{\alpha})$ depend on $\underline{\alpha}$. This is not the case for $k=1$, as proved in [14] and in [4]. Nor is it the case when we consider the point of view taken by Levesley [13], who showed that, for any real number $\xi$, any real $k$-tuple $\left(\xi_{1}, \ldots, \xi_{k}\right)$, and for any real numbers $v>k$ and $w>1 / k$, the Hausdorff dimensions of the sets

$$
\begin{array}{r}
\left\{\left(\alpha_{1}, \ldots, \alpha_{k}\right) \in \mathbb{R}^{k}:\left\|n_{1} \alpha_{1}+\cdots+n_{k} \alpha_{k}-\xi\right\|<\frac{1}{\left(\max _{1 \leq i \leq k}\left\{\left|n_{i}\right|\right\}\right)^{v}}\right. \\
\text { for infinitely many } \left.\left(n_{1}, \ldots, n_{k}\right) \in \mathbb{Z}^{k}\right\}
\end{array}
$$

and

$$
\left\{\left(\alpha_{1}, \ldots, \alpha_{k}\right) \in \mathbb{R}^{k}: \max _{1 \leq i \leq k}\left\{\left\|n \alpha_{i}-\xi_{i}\right\|\right\}<1 /|n|^{w}\right.
$$

for infinitely many $n \in \mathbb{Z}\}$

are equal to $k-1+(k+1) /(v+1)$ and $(k+1) /(w+1)$, respectively, independently of the real number $\xi$ and of the real $k$-tuple $\left(\xi_{1}, \ldots, \xi_{k}\right)$. To complement this result, we mention that, in the "doubly metric" case, Dodson [7] established that, for real numbers $v>k$ and $w>1 / k$, the Hausdorff dimensions of the sets

$$
\begin{array}{r}
\left\{\left(\alpha_{1}, \ldots, \alpha_{k}, \xi\right) \in \mathbb{R}^{k+1}:\left\|n_{1} \alpha_{1}+\cdots+n_{k} \alpha_{k}-\xi\right\|<\frac{1}{\left(\max _{1 \leq i \leq k}\left\{\left|n_{i}\right|\right\}\right)^{v}}\right. \\
\text { for infinitely many } \left.\left(n_{1}, \ldots, n_{k}\right) \in \mathbb{Z}^{k}\right\}
\end{array}
$$

and

$$
\begin{aligned}
& \left\{\left(\alpha_{1}, \ldots, \alpha_{k}, \xi_{1}, \ldots, \xi_{k}\right) \in \mathbb{R}^{k}: \max _{1 \leq i \leq k}\left\{\left\|n \alpha_{i}-\xi_{i}\right\|\right\}<1 /|n|^{w}\right. \\
& \text { for infinitely many } n \in \mathbb{Z}\}
\end{aligned}
$$

are equal to $k+(k+1) /(v+1)$ and $k+(k+1) /(w+1)$.

REMARK. In Theorems 1 and 2, we have given explicit constructions of real $k$-tuples with non-typical behaviour. A natural extension of our present work consists in studying the same questions, but for dependent $k$-tuples, that is, for instance, for $k$-tuples $\underline{\alpha}=\left(\alpha, \alpha^{2}, \ldots, \alpha^{k}\right)$, where $\alpha$ is a transcendental real number. It is known that, for almost all real numbers $\alpha$, the sets $\mathcal{V}_{v}(\underline{\alpha})$ and $\mathcal{W}_{w}(\underline{\alpha})$ satisfy $\operatorname{dim} \mathcal{V}_{v}(\underline{\alpha})=k / v$ and $\operatorname{dim} \mathcal{W}_{w}(\underline{\alpha})=1 / w$, for real numbers $v \geq k$ and $w \geq 1 / k$. Maybe, this statement even holds for all $\underline{\alpha}$ with $\alpha$ transcendental. We plan to investigate this problem in a further work. 
Notation. Except in Section 5 (that is, for the proof of Theorem 3), we use the following notation. Let $k \geq 2$ be an integer. We endow $\mathbb{R}^{k}$ with the supremum norm $|\cdot|$, and, for any $\underline{x}$ in $\mathbb{R}^{k}$, we set

$$
\|\underline{x}\|=\inf \left\{|\underline{x}-\underline{n}|: \underline{n} \in \mathbb{Z}^{k}\right\} .
$$

Clearly, $\|\cdot\|$ induces a distance on the $k$-dimensional torus $\mathbb{T}^{k}$, which we also denote by $\|\cdot\|$. If $Y$ is a subset of $\mathbb{R}^{k}$ and $\underline{x}$ is a point in $\mathbb{R}^{k}$, we denote by $d(\underline{x}, Y)$ the distance from $\underline{x}$ to $Y$, defined by

$$
d(\underline{x}, Y)=\inf \{\|\underline{x}-\underline{y}\|: \underline{y} \in Y\} .
$$

For the proof of Theorem 3, it appears to be more natural to endow $\mathbb{R}^{k}$ with the Euclidean norm, as specified at the beginning of Section 5.

3. Proof of Theorem 1. Let $v>1$ be real. We treat only the case $k=3$, since the case $k=2$ is much easier. Presumably, the argument works as well for any integer $k \geq 4$, but this is technically much more complicated. We first prove the existence of triples $\underline{\alpha}=\left(\alpha_{1}, \alpha_{2}, \alpha_{3}\right)$ for which the set

$$
\begin{array}{r}
\mathcal{V}_{v}^{\prime}(\underline{\alpha}):=\left\{\xi \in \mathbb{R}:\left\|n_{1} \alpha_{1}+n_{2} \alpha_{2}+n_{3} \alpha_{3}-\xi\right\|<\frac{1}{\left(\max _{1 \leq i \leq 3}\left\{n_{i}\right\}\right)^{v}}\right. \\
\text { for infinitely many } \left.\left(n_{1}, n_{2}, n_{3}\right) \in \mathbb{Z}_{\geq 1}^{3}\right\}
\end{array}
$$

has Hausdorff dimension $1 / v$. At the end of this section, we then briefly explain which adaptations should be made to get Theorem 1 .

Throughout the proof of Theorem 1 , we work on the circle $[0,1[$, and we denote by $\{\cdot\}$ the fractional part. In order to simplify the exposition we need to fix some notation.

Notation. Let $a$ and $b$ be real numbers. If $\{b\}>\{a\}$, then $[a, b]$ denotes the interval $[\{a\},\{b\}]$, otherwise $[a, b]$ denotes the union $[\{a\}, 1[\cup[0,\{b\}]$.

The proof of Theorem 1 essentially rests on the following elementary lemma.

Lemma 1. Let $a_{1}, \ldots, a_{n}$ be positive integers. Set $p_{n} / q_{n}=\left[0 ; a_{1}, \ldots, a_{n}\right]$. Let $\tau$ and $v \geq 1$ be real numbers. Let $\alpha=\left[0 ; a_{1}, \ldots, a_{n}, a_{n+1}, \ldots\right]$ be real and set $p_{n+1} / q_{n+1}=\left[0 ; a_{1}, \ldots, a_{n+1}\right]$. Then

and

$$
\bigcup_{j=0}^{q_{n+1}^{1 /(2 v)}}[j \alpha+\tau-\varepsilon, j \alpha+\tau+\varepsilon] \subset \bigcup_{j=0}^{q_{n}}[j \alpha+\tau-2 \varepsilon, j \alpha+\tau+2 \varepsilon]
$$

$$
\bigcup_{j=0}^{q_{n+1}^{1 /(2 v)}}[-j \alpha+\tau-\varepsilon,-j \alpha+\tau+\varepsilon] \subset \bigcup_{j=0}^{q_{n}}[-j \alpha+\tau-2 \varepsilon,-j \alpha+\tau+2 \varepsilon]
$$

for any real number $\varepsilon \geq q_{n+1}^{-1 / 2}$. 
Proof. The basic idea of the proof of Lemma 1 is the following. Let $q_{n}$ denote the denominator of the $n$th convergent of a real number $\alpha$. Then the points $\{\alpha\},\{2 \alpha\}, \ldots,\left\{q_{n} \alpha\right\}$ are well distributed in the unit interval $I$ : two consecutive points are at least $1 /\left(3 q_{n}\right)$ and at most $3 / q_{n}$ distant. If the $(n+1)$ th partial quotient $a_{n+1}$ of $\alpha$ is large, then for any integer $q$ with e.g. $q_{n} \leq q \leq q_{n+1}^{1 / 4}$ the point $\{q \alpha\}$ is very close to some point $\{j \alpha\}$ with $1 \leq j \leq q_{n}$. This means that, for $\ell$ not too small, an interval centred at $\{q \alpha\}$ of length $\ell$ is contained in the interval centred at $\{j \alpha\}$ of length $2 \ell$. We now turn to the proof.

Recall that, by the theory of continued fractions, we have $\left\|q_{n} \alpha\right\|<$ $1 / q_{n+1}$. Let $m$ be an integer with $q_{n} \leq m \leq q_{n+1}^{1 /(2 v)}$. Euclidean division of $m$ by $q_{n}$ yields non-negative integers $b$ and $r$ with $b \leq q_{n+1}^{1 /(2 v)}, 0 \leq r<q_{n}$, and $m=b q_{n}+r$. Consequently, we get

$$
\|\{m \alpha\}-\{r \alpha\}\| \leq q_{n+1}^{1 /(2 v)}\left\|q_{n} \alpha\right\| \leq q_{n+1}^{-1+1 /(2 v)} \leq q_{n+1}^{-1 / 2} \leq \varepsilon,
$$

by assumption. The lemma follows.

We construct inductively the sequences of partial quotients of the real numbers $\alpha_{1}, \alpha_{2}$ and $\alpha_{3}$, in such a way that we know a countable covering of the set $\mathcal{V}_{v}^{\prime}\left(\alpha_{1}, \alpha_{2}, \alpha_{3}\right)$. The basic idea consists in building numbers $\alpha_{1}, \alpha_{2}$, and $\alpha_{3}$ so that their sequences of denominators of convergents increase very rapidly and are far from one another.

For $j=1,2,3$, we denote by $\alpha_{j}=\left[0 ; a_{1, j}, a_{2, j}, \ldots\right]$ the continued fraction expansion of $\alpha_{j}$, and by $\left(p_{n, j} / q_{n, j}\right)_{n \geq 1}$ the sequence of its convergents. In the course of the proof, we adopt the following convention. For any set of triples of integers

$$
\widetilde{X}=\left\{\left(j_{1}, j_{2}, j_{3}\right): j_{i} \in J_{i}, i=1,2,3\right\},
$$

we define

$$
X=\bigcup_{\left(j_{1}, j_{2}, j_{3}\right) \in X}\left[\underline{j} \cdot \underline{\alpha}-\max _{1 \leq i \leq 3}\left\{j_{i}\right\}^{-v}, \underline{j} \cdot \underline{\alpha}+\max _{1 \leq i \leq 3}\left\{j_{i}\right\}^{-v}\right] .
$$

For brevity, we write $\underline{j} \cdot \underline{\alpha}=j_{1} \alpha_{1}+j_{2} \alpha_{2}+j_{3} \alpha_{3}$ for $\underline{j}=\left(j_{1}, j_{2}, j_{3}\right)$ and $\underline{\alpha}=\left(\alpha_{1}, \alpha_{2}, \alpha_{3}\right)$.

Assume that $a_{1,1}, \ldots, a_{n, 1}, a_{1,2}, \ldots, a_{n, 2}$ and $a_{1,3}, \ldots, a_{n, 3}$ have already been constructed and that we have

$$
q_{n, 1}>q_{n-1,3}^{2 v(n-1)^{2}}, \quad q_{n, 2}>q_{n, 1}^{2 v n^{2}}, \quad q_{n, 3}>q_{n, 2}^{2 v n^{2}} .
$$

It will be implicit that we take $a_{n+1,1}, a_{n+1,2}$, and $a_{n+1,3}$ large enough in order that $\left(I_{n+1}\right)$ holds. 
By Lemma 1 applied with $j_{2}$ and $j_{3}$ fixed, the union of intervals

$$
U_{n}^{(1)}:=\bigcup_{\substack{0 \leq j_{1} \leq j_{3}^{1 / n}, 0 \leq j_{2} \leq j_{3}^{1 / n} \\ q_{n, 1}^{n} \leq j_{3} \leq q_{n+1,1}^{1 /(2 v)}}}\left[\underline{j} \cdot \underline{\alpha}-4 j_{3}^{-v}, \underline{j} \cdot \underline{\alpha}+4 j_{3}^{-v}\right]
$$

contains

$$
V_{n}^{(1)}:=\bigcup_{\substack{0 \leq j_{1} \leq q_{n+1,1}^{1 /(2 v)}, 0 \leq j_{2} \leq j_{3}^{1 / n} \\ q_{n, 1}^{n} \leq j_{3} \leq q_{n+1,1}^{1 /(2 v)}}}\left[\underline{j} \cdot \underline{\alpha}-2 j_{3}^{-v}, \underline{j} \cdot \underline{\alpha}+2 j_{3}^{-v}\right]
$$

and thus as well the union

$$
X_{n}^{(1)}=\bigcup\left[\underline{j} \cdot \underline{\alpha}-\max _{1 \leq i \leq 3}\left\{j_{i}\right\}^{-v}, \underline{j} \cdot \underline{\alpha}+\max _{1 \leq i \leq 3}\left\{j_{i}\right\}^{-v}\right],
$$

taken over the set of triples

$\left(\widetilde{X}_{n}^{(1)}\right) \quad 0 \leq j_{1} \leq q_{n+1,1}^{1 /(2 v)}, \quad 0 \leq j_{2} \leq j_{3}^{1 / n}, \quad q_{n, 1}^{n} \leq j_{3} \leq q_{n+1,1}^{1 /(2 v)}$.

Furthermore, applying Lemma 1 to $V_{n}^{(1)}$ with $j_{1}$ and $j_{3}$ fixed, we see that $V_{n}^{(1)}$ contains

$$
\bigcup_{\substack{0 \leq j_{1} \leq q_{n+1,1}^{1 /(2 v)}, 0 \leq j_{2} \leq q_{n, 2}^{1 /(2 v)} \\ q_{n, 1}^{n} \leq j_{3} \leq q_{n, 2}^{1 / 2 v)}}}\left[\underline{j} \cdot \underline{\alpha}-j_{3}^{-v}, \underline{j} \cdot \underline{\alpha}+j_{3}^{-v}\right]
$$

and thus as well the union

$$
Y_{n}^{(1)}=\bigcup\left[\underline{j} \cdot \underline{\alpha}-\max _{1 \leq i \leq 3}\left\{j_{i}\right\}^{-v}, \underline{j} \cdot \underline{\alpha}+\max _{1 \leq i \leq 3}\left\{j_{i}\right\}^{-v}\right]
$$

taken over the set of triples

$$
\left(\tilde{Y}_{n}^{(1)}\right) \quad 0 \leq j_{1} \leq q_{n+1,1}^{1 /(2 v)}, \quad 0 \leq j_{2} \leq q_{n, 2}^{1 /(2 v)}, \quad q_{n, 1}^{n} \leq j_{3} \leq q_{n, 2}^{1 /(2 v)} .
$$

In the same way, reversing the rôles played by $j_{2}$ and $j_{3}$, we find that

$$
W_{n}^{(1)}:=\bigcup_{\substack{0 \leq j_{1} \leq j_{2}^{1 / n}, q_{n, 1}^{n} \leq j_{2} \leq q_{n+1,1}^{1 /(2 v)} \\ 0 \leq j_{3} \leq j_{2}^{1 / n}}}\left[\underline{j} \cdot \underline{\alpha}-4 j_{2}^{-v}, \underline{j} \cdot \underline{\alpha}+4 j_{2}^{-v}\right]
$$

contains the union of intervals

$$
Z_{n}^{(1)}=\bigcup\left[\underline{j} \cdot \underline{\alpha}-\max _{1 \leq i \leq 3}\left\{j_{i}\right\}^{-v}, \underline{j} \cdot \underline{\alpha}+\max _{1 \leq i \leq 3}\left\{j_{i}\right\}^{-v}\right]
$$

taken over the set of triples

$$
\left(\widetilde{Z}_{n}^{(1)}\right) \quad 0 \leq j_{1} \leq q_{n+1,1}^{1 /(2 v)}, \quad q_{n, 1}^{n} \leq j_{2} \leq q_{n+1,1}^{1 /(2 v)}, \quad 0 \leq j_{3} \leq j_{2}^{1 / n},
$$

and the union

$$
T_{n}^{(1)}=\bigcup\left[\underline{j} \cdot \underline{\alpha}-\max _{1 \leq i \leq 3}\left\{j_{i}\right\}^{-v}, \underline{j} \cdot \underline{\alpha}+\max _{1 \leq i \leq 3}\left\{j_{i}\right\}^{-v}\right]
$$


taken over the set of triples

$\left(\widetilde{T}_{n}^{(1)}\right) \quad 0 \leq j_{1} \leq q_{n+1,1}^{1 /(2 v)}, \quad q_{n, 1}^{n} \leq j_{2} \leq q_{n, 3}^{1 /(2 v)}, \quad 0 \leq j_{3} \leq q_{n, 3}^{1 /(2 v)}$.

Proceeding as above and letting 2 and 3 play the rôle of the index 1 , we further define finite unions of intervals $U_{n}^{(2)}, W_{n}^{(2)}, U_{n}^{(3)}$ and $W_{n}^{(3)}$ taken, respectively, over the triples defined by

$$
\begin{aligned}
0 & \leq j_{1} \leq j_{3}^{1 / n}, & 0 & \leq j_{2} \leq j_{3}^{1 / n}, & & q_{n, 2}^{n} \leq j_{3} \leq q_{n+1,2}^{1 /(2 v)}, \\
q_{n, 2}^{n} & \leq j_{1} \leq q_{n+1,2}^{1 /(2 v)}, & 0 & \leq j_{2} \leq j_{1}^{1 / n}, & 0 & \leq j_{3} \leq j_{1}^{1 / n} \\
q_{n, 3}^{n} & \leq j_{1} \leq q_{n+1,3}^{1 /(2 v)}, & 0 & \leq j_{2} \leq j_{1}^{1 / n}, & & \leq j_{3} \leq j_{1}^{1 / n} \\
0 & \leq j_{1} \leq j_{2}^{1 / n}, & q_{n, 3}^{n} & \leq j_{2} \leq q_{n+1,3}^{1 /(2 v)}, & & \leq j_{3} \leq j_{2}^{1 / n},
\end{aligned}
$$

and of length, respectively, $8 j_{3}^{-v}, 8 j_{1}^{-v}, 8 j_{1}^{-v}$, and $8 j_{2}^{-v}$.

By an application of Lemma 1 as above, they contain, respectively, the unions of intervals corresponding to the sets of triples:
$\left(\widetilde{X}_{n}^{(2)}\right)$
$0 \leq j_{1} \leq j_{3}^{1 / n}$,
$0 \leq j_{2} \leq q_{n+1,2}^{1 /(2 v)}$,
$q_{n, 2}^{n} \leq j_{3} \leq q_{n+1,2}^{1 /(2 v)}$,
$\left(\widetilde{Z}_{n}^{(2)} \quad q_{n, 2}^{n} \leq j_{1} \leq q_{n+1,2}^{1 /(2 v)}\right.$,
$0 \leq j_{2} \leq q_{n+1,2}^{1 /(2 v)}$,
$0 \leq j_{3} \leq j_{1}^{1 / n}$,
$\left(\widetilde{X}_{n}^{(3)}\right) \quad q_{n, 3}^{n} \leq j_{1} \leq q_{n+1,3}^{1 /(2 v)}$,
$0 \leq j_{2} \leq j_{1}^{1 / n}$,
$0 \leq j_{3} \leq q_{n+1,3}^{1 /(2 v)}$,
$\left(\widetilde{Z}_{n}^{(3)}\right)$
$0 \leq j_{1} \leq j_{2}^{1 / n}$,
$q_{n, 3}^{n} \leq j_{2} \leq q_{n+1,3}^{1 /(2 v)}$,
$0 \leq j_{3} \leq q_{n+1,3}^{1 /(2 v)}$.

They also contain, respectively, the intervals corresponding to the sets of triples:
$\left(\widetilde{Y}_{n}^{(2)}\right)$
$0 \leq j_{1} \leq q_{n+1,1}^{1 /(2 v)}$
$0 \leq j_{2} \leq q_{n+1,2}^{1 /(2 v)}$
$q_{n, 2}^{n} \leq j_{3} \leq q_{n+1,1}^{1 /(2 v)}$
$\left(\widetilde{T}_{n}^{(2)}\right)$
$q_{n, 2}^{n} \leq j_{1} \leq q_{n, 3}^{1 /(2 v)}$,
$0 \leq j_{2} \leq q_{n+1,2}^{1 /(2 v)}$
$0 \leq j_{3} \leq q_{n, 3}^{1 /(2 v)}$,
$\left(\tilde{Y}_{n}^{(3)}\right)$
$q_{n, 3}^{n} \leq j_{1} \leq q_{n+1,2}^{1 /(2 v)}$,
$0 \leq j_{2} \leq q_{n+1,2}^{1 /(2 v)}$,
$0 \leq j_{3} \leq q_{n+1,3}^{1 /(2 v)}$,
$\left(\widetilde{T}_{n}^{(3)}\right)$
$0 \leq j_{1} \leq q_{n+1,1}^{1 /(2 v)}$,
$q_{n, 3}^{n} \leq j_{2} \leq q_{n+1,1}^{1 /(2 v)}$,
$0 \leq j_{3} \leq q_{n+1,3}^{1 /(2 v)}$.

LEMma 2. The union

$$
\bigcup_{\substack{q_{n, 2}^{n} \leq j_{1} \leq q_{n+1,2}^{1 /(2 v)} \\ j_{1} \geq j_{2} \geq 0, j_{1} \geq j_{3} \geq 0}}\left[\underline{j} \cdot \underline{\alpha}-j_{1}^{-v}, \underline{j} \cdot \underline{\alpha}+j_{1}^{-v}\right]
$$

is included in $Z_{n}^{(2)} \cup Y_{n}^{(3)} \cup Y_{n}^{(2)} \cup T_{n}^{(2)}$. The union

$$
\bigcup_{\substack{q_{n+1,2}^{1 /(2 v)} \leq j_{1} \leq q_{n+1,2}^{n+1} \\ j_{1} \geq j_{2} \geq 0, j_{1} \geq j_{3} \geq 0}}\left[\underline{j} \cdot \underline{\alpha}-j_{1}^{-v}, \underline{j} \cdot \underline{\alpha}+j_{1}^{-v}\right]
$$


is included in $X_{n}^{(3)} \cup T_{n+1}^{(1)}$. The union

$$
\bigcup_{\substack{q_{n, 1}^{n} \leq j_{2} \leq q_{n+1,1}^{1 /(2 v)} \\ j_{2} \geq j_{1} \geq 0, j_{2} \geq j_{3} \geq 0}}\left[\underline{j} \cdot \underline{\alpha}-j_{2}^{-v}, \underline{j} \cdot \underline{\alpha}+j_{2}^{-v}\right]
$$

is included in $Z_{n}^{(1)} \cup T_{n}^{(3)} \cup Y_{n}^{(2)} \cup T_{n}^{(1)}$. The union

$$
\bigcup_{\substack{n+(2 v) \leq j_{2} \leq q_{n+1,1}^{n+1} \\ q_{n+1,1}^{1 /(1,1} \\ j_{2} \geq j_{1} \geq 0, j_{2} \geq j_{3} \geq 0}}\left[\underline{j} \cdot \underline{\alpha}-j_{2}^{-v}, \underline{j} \cdot \underline{\alpha}+j_{2}^{-v}\right]
$$

is included in $Y_{n}^{(3)} \cup Z_{n}^{(3)}$. The union

$$
\bigcup_{\substack{q_{n, 1}^{n} \leq j_{3} \leq q_{n+1,1}^{1 /(2 v)} \\ j_{3} \geq j_{2} \geq 0, j_{3} \geq j_{1} \geq 0}}\left[\underline{j} \cdot \underline{\alpha}-j_{3}^{-v}, \underline{j} \cdot \underline{\alpha}+j_{3}^{-v}\right]
$$

is included in $X_{n}^{(1)} \cup Y_{n}^{(2)} \cup T_{n}^{(1)} \cup Y_{n}^{(1)}$. The union

$$
\bigcup_{\substack{q_{n+1,1}^{1 /(2 v)} \leq j_{3} \leq q_{n+1,1}^{n+1} \\ j_{3} \geq j_{2} \geq 0, j_{3} \geq j_{1} \geq 0}}\left[\underline{j} \cdot \underline{\alpha}-j_{3}^{-v}, \underline{j} \cdot \underline{\alpha}+j_{3}^{-v}\right]
$$

is included in $X_{n}^{(2)} \cup Y_{n}^{(3)}$.

Proof. We content ourselves with checking the first assertion, since the proofs of the other five are similar. Observe that the set of triples $\left(j_{1}, j_{2}, j_{3}\right)$ such that $q_{n, 2}^{n} \leq j_{1} \leq q_{n+1,2}^{1 /(2 v)}, j_{1} \geq j_{2} \geq 0$, and $j_{1} \geq j_{3} \geq 0$ is contained in

$$
Z_{n}^{(2)} \cup\left\{\left(j_{1}, j_{2}, j_{3}\right): q_{n, 2}^{n} \leq j_{1} \leq q_{n+1,2}^{1 /(2 v)}, 0 \leq j_{2} \leq j_{1}, j_{1}^{1 / n} \leq j_{3} \leq j_{1}\right\} .
$$

The latter set is contained in

$$
Y_{n}^{(3)} \cup\left\{\left(j_{1}, j_{2}, j_{3}\right): q_{n, 2}^{n} \leq j_{1} \leq q_{n, 3}^{n}, 0 \leq j_{2} \leq j_{1}, j_{1}^{1 / n} \leq j_{3} \leq j_{1}\right\},
$$

hence, in the union

$$
Y_{n}^{(3)} \cup Y_{n}^{(2)} \cup\left\{\left(j_{1}, j_{2}, j_{3}\right): q_{n, 2}^{n} \leq j_{1} \leq q_{n, 3}^{n}, 0 \leq j_{2} \leq j_{1}, j_{1}^{1 / n} \leq j_{3} \leq q_{n, 2}^{n}\right\} .
$$

Since $j_{1}^{1 / n}>q_{n, 2}^{n}$ if $j_{1}>q_{n, 2}^{n^{2}}$, the last set of triples reduces to the set

$$
\left\{\left(j_{1}, j_{2}, j_{3}\right): q_{n, 2}^{n} \leq j_{1} \leq q_{n, 2}^{n^{2}}, 0 \leq j_{2} \leq j_{1}, j_{1}^{1 / n} \leq j_{3} \leq q_{n, 2}^{n}\right\} .
$$

Since $q_{n, 3}>q_{n, 2}^{2 v n^{2}}$, this is included in $T_{n}^{(2)}$. This completes the proof of the first assertion of the lemma.

Lemma 3. Let $E$ be a Borel subset of $\mathbb{R}^{k}$ and $\left\{U_{j}\right\}_{j \geq 1}$ be a countable family of subsets of $\mathbb{R}^{k}$ such that

$$
E \subset\left\{\xi \in \mathbb{R}: \xi \in U_{j} \text { for infinitely many } j \geq 1\right\}
$$


If $s$ is a real number such that

$$
\sum_{j \geq 1}\left(\operatorname{diam}\left(U_{j}\right)\right)^{s}<\infty
$$

then $\mathcal{H}^{s}(E)=0$ and $\operatorname{dim} E \leq s$.

Proof. This is the Hausdorff-Cantelli lemma (see e.g. [2]).

Lemma 4. For $j=1,2,3$, the sets $\lim \sup U_{n}^{(j)}$ and $\lim \sup W_{n}^{(j)}$ have Hausdorff dimension at most $1 / v$.

Proof. We only prove that $\operatorname{dim} \lim \sup U_{n}^{(1)} \leq 1 / v$. Let $s>1 / v$ be a real number. For any positive integer $n_{0}$, the $s$-measure of the set $\lim \sup U_{n}^{(1)}$ is at most

$$
\sum_{n \geq n_{0}} \sum_{j \geq q_{n, 1}^{n}}\left(j^{2 / n}+2\right) j^{-v s} .
$$

This double sum has the same behaviour as

$$
\sum_{n \geq n_{0}} q_{n, 1}^{2} q_{n, 1}^{n(1-v s)}
$$

which is convergent since $s>1 / v$. It then follows from Lemma 3 that $\operatorname{dim} \lim \sup U_{n}^{(1)} \leq 1 / v$.

Now, we complete the proof of the theorem. Let $\xi$ be in $\mathcal{V}_{v}^{\prime}\left(\alpha_{1}, \alpha_{2}, \alpha_{3}\right)$ with $\alpha_{1}, \alpha_{2}$ and $\alpha_{3}$ as above. Possibly after permuting $\alpha_{1}, \alpha_{2}$, and $\alpha_{3}, \xi$ belongs to infinitely many intervals

$$
\left[\underline{j} \cdot \underline{\alpha}-j_{1}^{-v}, \underline{j} \cdot \underline{\alpha}+j_{1}^{-v}\right]
$$

with $j_{1} \geq j_{2}$ and $j_{1} \geq j_{3}$. In view of Lemma 2 , this means that

$$
\xi \in \bigcup_{0 \leq j \leq 3} \lim \sup U_{n}^{(j)} \cup \bigcup_{0 \leq j \leq 3} \limsup W_{n}^{(j)} .
$$

The desired result follows from Lemma 4.

To go from the case of the sets $\mathcal{V}_{v}^{\prime}$ to that of the sets $\mathcal{V}_{v}$, we first have to slightly increase the size of the intervals; basically, we replace $j_{i}^{-v}$ by $j_{i}^{-v} \log j_{i}$. It is easily seen that Lemma 4 remains true with these slightly larger intervals. Furthermore, to go from the non-negative integers to all the rational integers, we simply observe that, as a consequence of the second part of Lemma 1 , the above discussion applies not only to $\left(\alpha_{1}, \alpha_{2}, \alpha_{3}\right)$, but to any of the eight triples $\left( \pm \alpha_{1}, \pm \alpha_{2}, \pm \alpha_{3}\right)$.

To conclude, it only remains to prove that $1, \alpha_{1}, \alpha_{2}$ and $\alpha_{3}$ are linearly independent over the rationals. Assume that there exist integers $A_{1}, \ldots, A_{4}$, not all zero, such that

$$
A_{1} \alpha_{1}+A_{2} \alpha_{2}+A_{3} \alpha_{3}+A_{4}=0 .
$$


For any positive integer $n$, we have

$$
A_{1} q_{n, 2} q_{n, 1} \alpha_{1}+A_{2} q_{n, 1} q_{n, 2} \alpha_{2}+A_{3} q_{n, 1} q_{n, 2} \alpha_{3}+q_{n, 1} q_{n, 2} A_{4}=0 .
$$

Classical results from the theory of continued fractions (see e.g. [16]) imply that

$$
\left\|A_{1} q_{n, 2} q_{n, 1} \alpha_{1}\right\| \ll q_{n, 2} q_{n+1,1}^{-1}
$$

and

$$
\left\|A_{2} q_{n, 1} q_{n, 2} \alpha_{2}\right\| \ll q_{n, 1} q_{n+2,1}^{-1} ;
$$

here and below, the numerical constants implied in $\ll$ depend only on $A_{1}, \ldots, A_{4}$. If $A_{3} \neq 0$, we get

$$
\left\|A_{3} q_{n, 1} q_{n, 2} \alpha_{3}\right\| \ll q_{n, 2} q_{n+1,1}^{-1} \ll\left(\left|A_{3}\right| q_{n, 1} q_{n, 2}\right)^{-2}
$$

for $n$ large enough. Then Legendre's theorem (see e.g. [16]) implies that $\left|A_{3}\right| q_{n, 1} q_{n, 2}$ is the denominator of a convergent to $\alpha_{3}$. This is a contradiction, since

$$
q_{n-1,3}<\left|A_{3}\right| q_{n, 1} q_{n, 2}<q_{n, 3}
$$

for $n$ large enough. Consequently, $A_{3}=0$ and we argue in a similar way to show that $A_{1}=A_{2}=A_{4}=0$, contrary to assumption. Thus, we have established that $1, \alpha_{1}, \alpha_{2}$ and $\alpha_{3}$ are linearly independent over the rationals. This completes the proof of the theorem.

4. Proof of Theorem 2. For simplicity we only do the proof for $k=3$. This is much more illustrative than the case $k=2$, and slightly less technical than the general case. At the end of this section, we indicate which (slight) changes are necessary in order to treat the case $k \geq 4$.

Replacing $\phi$ by the function $\widetilde{\phi}$ defined by $\widetilde{\phi}(n)=\sup _{j \geq n} \phi(j)$ if necessary, we may assume without any loss of generality that $\phi$ is non-increasing.

We aim to construct $\underline{\alpha}$ such that the Hausdorff dimension of

$$
\begin{aligned}
\mathcal{W}_{\phi}^{\prime}(\underline{\alpha})=\left\{\left(\xi_{1}, \xi_{2}, \xi_{3}\right) \in \mathbb{R}^{3}: \max _{1 \leq i \leq 3}\left\{\left\|n \alpha_{i}-\xi_{i}\right\|\right\}<\phi(|n|)\right. \\
\text { for infinitely many } \left.n \in \mathbb{Z}_{\geq 1}\right\}
\end{aligned}
$$

is less than or equal to 1 . Indeed, since $\mathcal{W}_{\phi}(\underline{\alpha})=\mathcal{W}_{\phi}^{\prime}(\underline{\alpha}) \cup\left(-\mathcal{W}_{\phi}^{\prime}(\underline{\alpha})\right)$, this implies the first statement of the theorem.

Let $(f(n))_{n \geq 0}$ be an increasing sequence of integers such that $f(0)=0$ and set

$$
\underline{\alpha}=\left(\sum_{n=0}^{\infty} 2^{-f(3 n)}, \sum_{n=0}^{\infty} 3^{-f(3 n+1)}, \sum_{n=0}^{\infty} 5^{-f(3 n+2)}\right) .
$$

We construct inductively the sequence $(f(n))_{n \geq 0}$ so that the corresponding triple $\underline{\alpha}$ satisfies $\operatorname{dim} \mathcal{W}_{\phi}^{\prime}(\underline{\alpha}) \leq 1$. 
In the following, $\theta$ denotes an element of $\mathbb{R}^{3}$ and $\Theta$ its projection on the torus $\mathbb{T}^{3}$. Consider the sequence $\left(\theta_{n}\right)_{n \geq 0}$ of triples defined by

$$
\begin{gathered}
\theta_{0}=\left(2^{-f(0)}, 0,0\right), \theta_{1}=\left(2^{-f(0)}, 3^{-f(1)}, 0\right), \theta_{2}=\left(2^{-f(0)}, 3^{-f(1)}, 5^{-f(2)}\right), \\
\theta_{3 n}=\left(\sum_{m=0}^{n} 2^{-f(3 m)}, \sum_{m=0}^{n-1} 3^{-f(3 m+1)}, \sum_{m=0}^{n-1} 5^{-f(3 m+2)}\right), \\
\theta_{3 n+1}=\left(\sum_{m=0}^{n} 2^{-f(3 m)}, \sum_{m=0}^{n} 3^{-f(3 m+1)}, \sum_{m=0}^{n-1} 5^{-f(3 m+2)}\right), \\
\theta_{3 n+2}=\left(\sum_{m=0}^{n} 2^{-f(3 m)}, \sum_{m=0}^{n} 3^{-f(3 m+1)}, \sum_{m=0}^{n} 5^{-f(3 m+2)}\right) .
\end{gathered}
$$

The sequence $\left(\theta_{n}\right)_{n \geq 0}$ converges to $\underline{\alpha}$.

For any integer $p \geq 2$, the projection $\Theta_{p}$ of $\theta_{p}$ is an element of $\mathbb{T}^{3}$ of finite order $Q_{p}=2^{f\left(p_{0}\right)} 3^{f\left(p_{1}\right)} 5^{f\left(p_{2}\right)}$, where $p_{i}$ denotes the largest integer $\leq p$ of the form $p_{i}=3 m+i$.

Observe that all the exponents $f(m)$ occurring in $\eta_{n}:=\alpha-\theta_{n}$ are strictly larger than $f(n)$. Thus, we can choose the sequence $(f(n))_{n \geq 0}$ such that $\left|\eta_{n}\right|$ decreases arbitrarily rapidly to 0 . Further, we check that

$$
\mathbb{Z} \theta_{p}+\mathbb{Z}^{3}=\mathbb{Z}\left(2^{-f\left(p_{0}\right)}, 0,0\right)+\mathbb{Z}\left(0,3^{-f\left(p_{1}\right)}, 0\right)+\mathbb{Z}\left(0,0,5^{-f\left(p_{2}\right)}\right)=: \Gamma_{p}
$$

for any integer $p \geq 2$. Therefore, the distance of any point $x$ of $\mathbb{R}^{3}$ to $\Gamma_{p}$ goes to zero as $p$ tends to infinity. For every integer $q$ in $\left\{0, \ldots, Q_{p}\right\}$, we have

$$
d\left(q \theta_{p}, q \underline{\alpha}\right) \leq q\left|\eta_{p}\right| \leq Q_{p}\left|\eta_{p}\right| .
$$

Thus, the distance of any point $x$ in $\mathbb{R}^{3}$ to $\mathbb{Z} \underline{\alpha}+\mathbb{Z}^{3}$ is at most $Q_{p}\left|\eta_{p}\right|+d\left(x, \Gamma_{p}\right)$. Consequently, $\mathbb{Z} \underline{\alpha}+\mathbb{Z}^{3}$ is everywhere dense in $\mathbb{R}^{3}$. This shows that 1 and the three coordinates of $\underline{\alpha}$ are linearly independent over the rationals.

The proof of Theorem 2 rests on the next three lemmas.

Lemma 5. For any integer $p \geq 2$, the projection $G_{p}$ of $\Gamma_{p}$ in $\mathbb{T}^{3}$ is contained in a set $D_{p}$ which is a union of segments of total length

$$
L_{p}= \begin{cases}3^{f(p-2)} 5^{f(p-1)} & \text { if } p=3 n, \\ 5^{f(p-2)} 2^{f(p-1)} & \text { if } p=3 n+1, \\ 2^{f(p-2)} 3^{f(p-1)} & \text { if } p=3 n+2 .\end{cases}
$$

Furthermore, all these segments are of length 1.

Proof. We only treat the case $p=3 n$, since the other two are similar. We observe that $G_{p}$ is included in the projection of the segments $[0,1] \times$ $\left\{\left(a 3^{-f(p-2)}, b 5^{-f(p-1)}\right)\right\}$ for $a=1, \ldots, 3^{f(p-2)}$ and $b=1, \ldots, 5^{f(p-1)}$. 
Lemma 6 . The sequence $(f(n))_{n \geq 0}$ may be chosen in such a way that there exists a sequence $\left(P_{n}\right)_{n \geq 0}$ of integers satisfying

$$
Q_{n}<P_{n}<Q_{n+1}
$$

for any integer $n \geq 1$.

Proof. We proceed by induction. Assume that $f(0), \ldots, f(n)$ and $P_{0}, \ldots$ $\ldots, P_{n-1}$ are constructed. Since $L_{n+1}$ depends only on $f(0), \ldots, f(n)$, we can choose an integer $P_{n}>Q_{n}$ such that $\phi\left(P_{n}\right) \leq e^{-L_{n+1}}$. Taking $f(n+1)$ large, we get (2), $\phi\left(Q_{n+1}\right) \leq e^{-L_{n+1}}$, and $Q_{n+1}>P_{n}$.

LEMMA 7. Let $\left(\varepsilon_{n}\right)_{n \geq 1}$ be a sequence of positive real numbers which tends to 0 . If $\left(E_{n}\right)_{n \geq 1}$ is a sequence of sets such that $\sum_{n \geq 1} \mathcal{H}_{\varepsilon_{n}}^{s}\left(E_{n}\right)<\infty$, then

$$
\mathcal{H}^{s}\left(\limsup _{n \rightarrow \infty} E_{n}\right)=0,
$$

and the Hausdorff dimension of $\lim \sup _{n \rightarrow \infty} E_{n}$ is at most $s$.

Proof. This is an easy consequence of the Hausdorff-Cantelli lemma (see e.g. $[2$, p. 68$])$. Since

$$
\mathcal{H}_{\delta}^{s}\left(\limsup _{n \rightarrow \infty} E_{n}\right) \leq \sum_{n: \varepsilon_{n} \leq \delta} \mathcal{H}_{\varepsilon_{n}}^{s}\left(E_{n}\right)
$$

for any $\delta>0$, we get $\mathcal{H}^{s}\left(\lim \sup _{n \rightarrow \infty} E_{n}\right)=0$.

For a subset $E$ of $\mathbb{T}^{3}$ and a positive real number $r$, we put

$$
V(E, r)=\left\{x \in \mathbb{T}^{3}: d(x, E) \leq r\right\} .
$$

Further, for a positive integer $q$, we set $E_{q}=\{0, \Theta, 2 \Theta, \ldots, q \Theta\}$.

Since $Q_{n}<P_{n}<Q_{n+1}$ and $\phi$ is non-increasing, $\mathcal{W}_{\phi}^{\prime}(\underline{\alpha})$ is included in

$$
\limsup _{n \rightarrow \infty} V\left(E_{P_{n}}, \phi\left(Q_{n}\right)\right) \cup \limsup _{n \rightarrow \infty} V\left(E_{Q_{n+1}}, \phi\left(P_{n}\right)\right) .
$$

To establish that the Hausdorff dimension of $\mathcal{W}_{\phi}^{\prime}(\underline{\alpha})$ is at most 1 , it is sufficient, by Lemma 7 , to prove that, for any $s>1$, there exist two sequences $\left(\varepsilon_{n}\right)_{n \geq 1}$ and $\left(\varepsilon_{n}^{\prime}\right)_{n \geq 1}$ which decrease to 0 and are such that the series

$$
\sum_{n} \mathcal{H}_{\varepsilon_{n}}^{s}\left(V\left(E_{P_{n}}, \phi\left(Q_{n}\right)\right)\right) \text { and } \sum_{n} \mathcal{H}_{\varepsilon_{n}^{\prime}}^{s}\left(V\left(E_{Q_{n+1}}, \phi\left(P_{n}\right)\right)\right)
$$

converge.

Let $q \leq P_{n}$ be a positive integer. Euclidean division of $q$ by $Q_{n}$ yields integers $l$ and $a$ such that $q=l Q_{n}+a$ and $0 \leq a<Q_{n}$. Therefore,

$$
\left\|q \Theta-a \Theta_{n}\right\|=\left\|\left(l Q_{n}+a\right)\left(\Theta_{n}+\eta_{n}\right)-a \Theta_{n}\right\| \leq\left|\left(l Q_{n}+a\right) \eta_{n}\right| \leq P_{n}\left|\eta_{n}\right| .
$$


Furthermore, by (2), we get $P_{n}\left|\eta_{n}\right| \leq \phi\left(Q_{n}\right)$, thus

$$
E_{P_{n}} \subset V\left(\left\{0, \Theta_{n}, \ldots,\left(Q_{n}-1\right) \Theta_{n}\right\}, \phi\left(Q_{n}\right)\right) .
$$

It follows that

$$
V\left(E_{P_{n}}, \phi\left(Q_{n}\right)\right) \subset V\left(\left\{0, \Theta_{n}, \ldots,\left(Q_{n}-1\right) \Theta_{n}\right\}, 2 \phi\left(Q_{n}\right)\right),
$$

thus, by Lemma 5 , we get

$$
V\left(E_{P_{n}}, \phi\left(Q_{n}\right)\right) \subset V\left(D_{n}, 2 \phi\left(Q_{n}\right)\right) .
$$

Let $s>1$ be a real number and $n$ be a positive integer. Throughout the remaining part of the proof, « means that there is an implied absolute positive constant. Setting $\varepsilon_{n}=\phi\left(Q_{n}\right)$, we have

$$
\mathcal{H}_{\varepsilon_{n}}^{s}\left(V\left(D_{n}, 2 \phi\left(Q_{n}\right)\right)\right) \ll \frac{\text { length of } D_{n}}{\varepsilon_{n}} \cdot \varepsilon_{n}^{s} \ll L_{n} \phi\left(Q_{n}\right)^{s-1} .
$$

It then follows from (1) that

$$
\mathcal{H}_{\varepsilon_{n}}^{s}\left(V\left(E_{P_{n}}, \phi\left(Q_{n}\right)\right)\right) \ll L_{n} e^{-(s-1) L_{n}} .
$$

This last inequality shows that the series $\sum_{n} \mathcal{H}_{\varepsilon_{n}}^{s}\left(V\left(E_{P_{n}}, Q_{n}^{-v}\right)\right)$ converges. For the other series, set $\varepsilon_{n}^{\prime}=\phi\left(P_{n}\right)$. For $q \leq Q_{n+1}$, we have

$$
\left\|q \Theta-q \Theta_{n+1}\right\| \leq Q_{n+1}\left|\eta_{n+1}\right|,
$$

and then, by (2),

$$
\left\|q \Theta-q \Theta_{n+1}\right\| \leq \phi\left(P_{n}\right)
$$

Therefore, we get

$$
\begin{aligned}
V\left(E_{Q_{n+1}}, \phi\left(P_{n}\right)\right) & \subset V\left(\left\{0, \Theta_{n+1}, \ldots, Q_{n+1} \Theta_{n+1}\right\}, 2 \phi\left(P_{n}\right)\right) \\
& \subset V\left(D_{n+1}, 2 \phi\left(P_{n}\right)\right)
\end{aligned}
$$

and

$$
\mathcal{H}_{\varepsilon_{n}^{\prime}}^{s}\left(V\left(E_{Q_{n+1}}, \phi\left(P_{n}\right)\right)\right) \ll \frac{\text { length of } D_{n+1}}{\varepsilon_{n}^{\prime}} \cdot \varepsilon_{n}^{\prime s} \ll L_{n+1} \phi\left(P_{n}\right)^{s-1} .
$$

Finally, by (1), we obtain

$$
\mathcal{H}_{\varepsilon_{n}^{\prime}}^{s}\left(V\left(E_{Q_{n+1}}, P_{n}^{-v}\right)\right) \ll L_{n+1} e^{-(s-1) L_{n+1}},
$$

which shows that the series $\sum_{n} \mathcal{H}_{\varepsilon_{n}^{\prime}}^{s}\left(V\left(E_{P_{n}}, \phi\left(Q_{n}\right)\right)\right)$ converges.

Consequently, the Hausdorff dimension of $\mathcal{W}_{\phi}^{\prime}(\underline{\alpha})$ is at most 1.

Taking for $\phi$ a function tending to 0 more slowly than any function $x \mapsto x^{-w}$ with $w>0$, we get the second statement of the theorem.

To conclude, we briefly explain how to deal with the general case. For any integer $k \geq 2$, we choose the first $k$ prime numbers $p_{1}=2, p_{2}=3, \ldots, p_{k}$ and we set

$$
\underline{\alpha}=\left(\sum_{n=0}^{\infty} p_{1}^{f(k n)}, \sum_{n=0}^{\infty} p_{2}^{f(k n+1)}, \ldots, \sum_{n=0}^{\infty} p_{k}^{f(k n+k-1)}\right) .
$$


We define a sequence $\left(\theta_{n}\right)_{n \geq 0}$ in the same way as above, that is, such that for any $i$ and $j$ in $\{0, \ldots, k-1\}$, the $j$ th coordinate of $\theta_{k n+i}$ is

$$
\begin{array}{ll}
\sum_{m=0}^{n} p_{j}^{f(k n+j-1)} & \text { if } j \leq i+1, \\
\sum_{m=0}^{n-1} p_{j}^{f(k n+j-1)} & \text { if } j>i+1 .
\end{array}
$$

The proof goes exactly as in the case $k=3$. The main point is that in Lemma 6 the length $L_{p}$ depends only on $f(0), \ldots, f(p-1)$.

5. Proof of Theorem 3. In this section, we use the following notation. Let $k \geq 2$ be an integer. We endow $\mathbb{R}^{k}$ with the Euclidean norm $|\cdot|_{2}$, and, for any $\underline{x}$ in $\mathbb{R}^{k}$, we set

$$
\|\underline{x}\|_{2}=\inf \left\{|\underline{x}-\underline{n}|_{2}: \underline{n} \in \mathbb{Z}^{k}\right\} .
$$

Clearly, $\|\cdot\|_{2}$ induces a distance on the $k$-dimensional torus $\mathbb{T}^{k}$, which we also denote by $\|\cdot\|_{2}$. If $Y$ is a subset of $\mathbb{R}^{k}$ and $\underline{x}$ is a point in $\mathbb{R}^{k}$, we denote by $d_{2}(\underline{x}, Y)$ the distance from $\underline{x}$ to $Y$, defined by

$$
d_{2}(\underline{x}, Y)=\inf \left\{\|\underline{x}-\underline{y}\|_{2}: \underline{y} \in Y\right\} .
$$

We could as well have worked with the supremum norm, as in the rest of the paper; however, since geometric arguments are applied in the present case, it seems to us more natural to use the Euclidean norm.

Furthermore, throughout this section, the constants implied by $\asymp, \ll$ and $\gg$ depend only on the dimension $k$.

First, we introduce the notions of best approximation in $\mathbb{R}^{k}$ and in the torus $\mathbb{T}^{k}$ (see e.g. [12]). These are needed to establish Lemma 9. As in Section 4 , if $\theta$ is an element of $\mathbb{R}^{k}$, we denote by $\Theta$ its projection on the torus $\mathbb{T}^{k}$.

Definition 1. Let $\Theta$ be in $\mathbb{T}^{k}$. A positive integer $q$ is a best approximation of $\Theta$ if we have $\|p \Theta\|_{2}>\|q \Theta\|_{2}$ for every integer $p$ with $0<p<q$. Let $\theta$ be in $\mathbb{R}^{k}$. A positive integer $q$ is a best approximation of $\theta$ if it is a best approximation of $\Theta$.

Let $\theta$ be in $\mathbb{R}^{k}$. Arranging the set of best approximations of $\theta$ in increasing order, we get an increasing sequence $\left(q_{n}\right)_{n \geq 0}$ of positive integers starting with $q_{0}=1$. For any positive integer $n$, let $\varepsilon_{n}$ be the vector in $\mathbb{R}^{k}$ and $P_{n}$ be the integer $k$-tuple such that

$$
q_{n} \theta=P_{n}+\varepsilon_{n} \quad \text { and } \quad\left|\varepsilon_{n}\right|_{2}=\left\|q_{n} \Theta\right\|_{2} .
$$

Put

$$
\theta_{n}=\theta-\frac{1}{q_{n}} \varepsilon_{n}=\frac{1}{q_{n}} P_{n} \quad \text { and } \quad r_{n}=\left|\varepsilon_{n}\right|_{2}=\left\|q_{n} \theta\right\|_{2} .
$$


Then $\theta_{n}$ is the rational approximation of $\theta$ corresponding to the best approximation $q_{n}$, and obviously $\left\|q_{n} \theta_{n}\right\|_{2}=0$. We consider the lattice

$$
\Lambda_{n}:=\mathbb{Z}^{k}+\mathbb{Z} \theta_{n},
$$

which is included in $\mathbb{Q}^{k}$, since $\theta_{n}$ has rational coordinates. We denote by $\lambda_{1, n}, \ldots, \lambda_{k, n}$ the successive minima of $\Lambda_{n}$.

Lemma 8. The subgroup $\left\langle\Theta_{n}\right\rangle$ of $\mathbb{T}^{k}$ generated by $\Theta_{n}$ has exactly $q_{n}$ elements, that is, $k \Theta_{n}$ is non-zero for any $k=1, \ldots, q_{n}-1$. Furthermore, for any $p=0,1, \ldots, q_{n}-1$, we have

$$
\left\|p \theta-p \theta_{n}\right\| \leq r_{n}
$$

Moreover, the lattice $\Lambda_{n}$ has determinant $1 / q_{n}$ and its first minimum $\lambda_{1, n}$ satisfies

$$
2 r_{n-1} \geq \lambda_{1, n} \geq r_{n-1} / 2 .
$$

Proof. This follows from [6, Lemme 2], since, with the notation of [6], the first minimum of $\Lambda_{n}$ is equal to $d\left(0,\left\langle\Theta_{n}\right\rangle \backslash\{0\}\right)$ and therefore to $r\left(\left\langle\Theta_{n}\right\rangle\right)$.

Lemma 9. The last minimum of $\Lambda_{n}$ tends to 0 as $n$ tends to infinity. The product $q_{n} r_{n-1}$ tends to infinity with $n$.

Proof. For any positive integer $q$, set $F_{q}=\{0, \theta, \ldots, q \theta\}+\mathbb{Z}^{k}$. By Lemma 8, the distance of each point of $F_{q_{n}-1}$ to $\Lambda_{n}$ is less than $r_{n}$, and

$$
\max _{x \in \mathbb{R}^{k}} d_{2}\left(x, \Lambda_{n}\right) \asymp \lambda_{k, n} .
$$

Consequently, we have

$$
\lambda_{k, n} \ll \max _{x \in \mathbb{R}^{k}} d_{2}\left(x, F_{q_{n}-1}\right)+r_{n},
$$

and, since $\mathbb{Z} \theta+\mathbb{Z}^{k}$ is dense in $\mathbb{R}^{k}$, we get

$$
\lim _{n \rightarrow \infty} \max _{x \in \mathbb{R}^{k}} d_{2}\left(x, F_{q_{n}-1}\right)=0 \text { and } \lim _{n \rightarrow \infty} \lambda_{k, n}=0,
$$

thus the last minimum of $\Lambda_{n}$ tends to 0 as $n$ tends to infinity.

By Minkowski's second theorem on successive minima (see e.g. [5, p. 156]), we have

$$
\operatorname{det} \Lambda_{n}=1 / q_{n} \asymp \lambda_{1, n} \lambda_{2, n} \cdots \lambda_{k, n} \leq \lambda_{1, n} \lambda_{k, n}^{k-1} .
$$

Combined with Lemma 8 , this gives

$$
\frac{1}{q_{n} r_{n-1}} \asymp \frac{1}{q_{n} \lambda_{1, n}} \leq \lambda_{k, n}^{k-1} .
$$

Since $k \geq 2$, it follows from (3) and (4) that $q_{n} r_{n-1}$ tends to infinity with $n$, as asserted.

After these preliminaries, we turn to the proof of Theorem 3 . Let $w \geq 1$ be a real number and $s$ be any real number in $] 0,1 / w\left[\right.$. Let $\underline{\alpha}=\left(\alpha_{1}, \ldots, \alpha_{k}\right)$ 
with $1, \alpha_{1}, \ldots, \alpha_{k}$ linearly independent over the rationals. We shall prove that $\mathcal{W}_{w}(\underline{\alpha})$ contains a Cantor-type set $\mathcal{K}$ whose Hausdorff dimension is greater than $s$. By the mass distribution principle (see e.g. [9, p. 24]; this is also called the Frostman lemma), it is sufficient to construct a probability measure $\mu$ on $\mathcal{K}$ such that

$$
\lim _{r \rightarrow 0} \frac{\mu(B(x, r))}{r^{s}}=0
$$

for all $x$ in $\mathcal{K}$. We divide our inductive construction into 6 steps.

STEP 1. For any positive integer $q$, set $F_{q}=\{0, \underline{\alpha}, \ldots, q \underline{\alpha}\}+\mathbb{Z}^{k}$ and $E_{q}=F_{q} \cap[0,1]^{k}$. Let $\left(q_{n}\right)_{n \geq 0}$ denote the sequence of best approximations of $\underline{\alpha}$ and, for any positive integer $n$, put

$$
\Lambda_{n}=\mathbb{Z}^{k}+\frac{\mathbb{Z}}{q_{n}} P_{n},
$$

where $P_{n}$ is the point of the lattice $\mathbb{Z}^{k}$ for which $q_{n} \underline{\alpha}-P_{n}$ is minimal.

For any positive integer $n$, put

$$
A_{n}=\left\{x \in[0,1]^{k}: d_{2}\left(x, E_{q_{n}-1}\right) \leq 1 / q_{n}^{w}\right\} .
$$

Let $\left(n_{j}\right)_{j \geq 1}$ be an increasing sequence of positive integers, which will be chosen in Step 6, and put

$$
\mathcal{K}=\bigcap_{j \geq 1} A_{n_{j}} .
$$

First, we observe that $\mathcal{K}$ is a Cantor-type set. Indeed, the sets $A_{n}$ are made up of closed balls and, by the definition of best approximation, the distance between the centres of two balls composing $A_{n}$ is at least $r_{n-1}$. Furthermore, it follows from Lemma 9 that, for $n$ sufficiently large, $A_{n}$ is a disjoint union of balls of the same radius.

To simplify the notation, for any integer $j \geq 1$, we put

$$
Q_{j}=q_{n_{j}}, \quad \Lambda^{j}=\Lambda_{n_{j}}, \quad \varrho_{j}=r_{n_{j}-1},
$$

and

$$
K_{j}=\bigcap_{1 \leq p \leq j} A_{n_{p}}
$$

STEP 2. Since $1, \alpha_{1}, \ldots, \alpha_{k}$ are linearly independent over the rationals, the sequence $(m \underline{\alpha})_{m \geq 1}$ is uniformly distributed in the torus $\mathbb{T}^{k}$. Thus, we may select $n_{j+1}$ sufficiently large in order that each ball of $K_{j}$ contains a number

$$
N \in\left[c_{k} Q_{j+1} Q_{j}^{-w k} / 2,2 c_{k} Q_{j+1} Q_{j}^{-w k}\right]
$$

of balls of $K_{j+1}$, where $c_{k}$ denotes the volume of the unit ball of $\mathbb{R}^{k}$. Dropping some of these balls if necessary, we can suppose that each of the balls of $K_{j}$ 
contains exactly $N_{j}=\left[c_{k} Q_{j+1} Q_{j}^{-w k} / 2\right]$ balls of $K_{j+1}$. Let $\mathcal{E}_{j}$ denote the set of centres of the balls composing $K_{j}$.

We define inductively a sequence of discrete probability measures $\left(\mu_{j}\right)_{j \geq 1}$ such that, for any $j \geq 1$, we have:

(i) the support of $\mu_{j}$ is equal to $\mathcal{E}_{j}$;

(ii) all the points of $\mathcal{E}_{j}$ have the same mass $m_{j}$.

Since the $\mu_{j}$ are probability measures, we get

$$
m_{j+1}=\frac{m_{j}}{N_{j}} \asymp m_{j} \frac{2}{c_{k}} Q_{j}^{w k} Q_{j+1}^{-1} .
$$

The sequence $\left(\mu_{j}\right)_{j \geq 1}$ weakly converges to a probability measure $\mu$ whose support is contained in $\mathcal{K}$.

STEP 3. Let $x$ be in $\mathcal{K}$. Let $j \geq 1$ be an integer. We wish to estimate $\mu(B(x, r)) r^{-s}$ for $r$ in $\left[\frac{1}{2} Q_{j+1}^{-w}, \frac{1}{2} Q_{j}^{-w}\right]$. There is a point $P_{j}$ in $E_{Q_{j}-1}$ such that the ball $B_{j}=B\left(P_{j}, Q_{j}^{-w}\right)$ of $K_{j}$ contains $x$. Let $\mathcal{C}_{j}$ be the set of centres of balls of $K_{j+1}$ included in $B_{j}$. Since all balls of $K_{j+1}$ have the same radius $Q_{j+1}^{-w}$, we have

$$
\mu(B(x, r)) \leq \mu_{j+1}\left(B\left(x, r+Q_{j+1}^{-w}\right)\right) .
$$

Therefore, in order to estimate $\mu(B(x, r))$, it is sufficient to count the number of points of $\mathcal{C}_{j}$ lying in $B\left(x, r+Q_{j+1}^{-w}\right)$.

We begin with an obvious upper bound. By the definition of $\varrho_{j+1}$, the distance between any two points of $E_{Q_{j+1}-1}$ is at least $\varrho_{j+1}$ and since $\mathcal{C}_{j}$ is contained in $E_{Q_{j+1}-1}$, we get

$$
\operatorname{card} B(x, r) \cap \mathcal{C}_{j} \ll \max \left\{1,\left(r / \varrho_{j+1}\right)^{k}\right\} .
$$

When $r / \varrho_{j+1}$ is large, this estimate is useless and a sharper upper bound is required. Since the set $E_{Q_{j+1}-1}$ is close to the lattice $\Lambda^{j+1}$, we begin by counting the points of $\Lambda^{j+1} \cap B(x, r)$.

STEP 4. Let $\left(e_{1}, \ldots, e_{k}\right)$ be a reduced basis of $\Lambda^{j+1}$. By "reduced", we mean (see [1]) that the following two properties hold true:

$$
\begin{gathered}
\left|e_{1}\right|_{2} \leq \cdots \leq\left|e_{k}\right|_{2}, \\
\sin \left(\angle\left(e_{i}, V_{i}\right)\right) \geq(\sqrt{3} / 2)^{k} \quad \text { for } i=1, \ldots, k,
\end{gathered}
$$

where $V_{i}=\operatorname{span}\left(e_{1}, \ldots, e_{i-1}, e_{i+1}, \ldots, e_{k}\right)$. This last inequality implies that, for any real numbers $t_{1}, \ldots, t_{k}$, we have

$$
\left|\sum_{i=1}^{k} t_{i} e_{i}\right|_{2} \geq(\sqrt{3} / 2)^{k} \max \left\{\left|t_{i}\right| \cdot\left|e_{i}\right|_{2}: i=1, \ldots, k\right\} .
$$

Let $y=\sum_{i=1}^{k} a_{i} e_{i}$ be in $\Lambda^{j+1} \cap B(0, r)$. Assume first that there exists $i$ with $1 \leq i \leq k-1$ such that $r$ is in $\left[(\sqrt{3} / 2)^{k}\left|e_{i}\right|_{2},(\sqrt{3} / 2)^{k}\left|e_{i+1}\right|_{2}\right.$ [. Then, 
by (6), we have

$$
\left|a_{j}\right| \leq \frac{r}{(\sqrt{3} / 2)^{k}\left|e_{j}\right|_{2}}, \quad j=1, \ldots, i,
$$

and, since the $a_{j}$ 's are integers,

$$
a_{j}=0, \quad j=i+1, \ldots, k .
$$

Therefore, we get

$$
\operatorname{card} \Lambda^{j+1} \cap B(0, r) \ll \frac{r^{i}}{\left|e_{1}\right|_{2} \cdots\left|e_{i}\right|_{2}} .
$$

Assume now that $r \geq(\sqrt{3} / 2)^{k}\left|e_{k}\right|_{2}$. Then we get the upper bound

$$
\operatorname{card} \Lambda^{j+1} \cap B(0, r) \ll \frac{r^{k}}{\left|e_{1}\right|_{2} \cdots\left|e_{k}\right|_{2}} \ll \frac{r^{k}}{\operatorname{det} \Lambda^{j+1}}=r^{k} Q_{j+1},
$$

by Lemma 8 . Furthermore, we observe that, for any $x$ in $\mathbb{R}^{k}$, we have

$$
\operatorname{card} \Lambda^{j+1} \cap B(z, r) \leq \operatorname{card} \Lambda^{j+1} \cap B(0,2 r) .
$$

STEP 5. Thanks to (5), (7) and (8), we are now able to bound $\mu(B(x, r)) r^{-s}$ from above. We distinguish three cases.

Assume first that $r$ is in $\left[\frac{1}{2} Q_{j+1}^{-w},\left|e_{1}\right|_{2}\right]$. From the "obvious" estimate (5), we get

$$
\begin{aligned}
\frac{\mu(B(x, r))}{r^{s}} & \leq \frac{\mu_{j+1}\left(B\left(x, r+Q_{j+1}^{-w}\right)\right)}{r^{s}} \\
& \ll m_{j+1} \max \left\{1,\left(2 r /\left|e_{1}\right|_{2}\right)^{k}\right\} r^{-s} \\
& \ll m_{j+1} r^{-s} \ll m_{j+1} Q_{j+1}^{s w} \ll m_{j} Q_{j}^{w k} Q_{j+1}^{w s-1} .
\end{aligned}
$$

Assume now that $r \geq\left|e_{1}\right|_{2}$ and that $r$ is in

$$
\left[(\sqrt{3} / 2)^{k}\left|e_{i}\right|_{2} / 6,(\sqrt{3} / 2)^{k}\left|e_{i+1}\right|_{2} / 6\right]
$$

for some $i=1, \ldots, k-1$. Then we infer from (7) that

$$
\begin{aligned}
\frac{\mu(B(x, r))}{r^{s}} & \leq \frac{\mu_{j+1}\left(B\left(x, r+Q_{j+1}^{-w}\right)\right)}{r^{s}} \\
& \ll \frac{m_{j+1} \operatorname{card} \Lambda^{j+1} \cap B\left(x, r+Q_{j+1}^{-w}+\left|e_{1}\right|_{2}\right)}{r^{s}} \\
& \ll \frac{m_{j+1}}{r^{s}} \frac{r^{i}}{\left|e_{1}\right|_{2} \cdots\left|e_{i}\right|_{2}}=\frac{m_{j+1}}{r^{s}} \frac{r^{i}\left|e_{i+1}\right|_{2} \cdots\left|e_{k}\right|_{2}}{\left|e_{1}\right|_{2} \cdots\left|e_{k}\right|_{2}} .
\end{aligned}
$$


Since $\left|e_{1}\right|_{2} \cdots\left|e_{k}\right|_{2} \geq \operatorname{det} \Lambda^{j+1}=1 / Q_{j+1}$, from (9) we get

$$
\begin{aligned}
\frac{\mu(B(x, r))}{r^{s}} & \ll m_{j+1} r^{i-s} Q_{j+1}\left|e_{i+1}\right|_{2} \cdots\left|e_{k}\right|_{2} \\
& \ll m_{j} \frac{Q_{j}^{w k}}{Q_{j+1}}\left|e_{i+1}\right|_{2}^{i-s} Q_{j+1}\left|e_{i+1}\right|_{2} \cdots\left|e_{k}\right|_{2} \\
& \ll m_{j} Q_{j}^{w k}\left|e_{k}\right|_{2}^{k-s} \\
& \ll m_{j} Q_{j}^{w k} \cdot\left(\text { last minimum of } \Lambda^{j+1}\right)^{k-s} .
\end{aligned}
$$

Indeed, for each $i,\left|e_{i}\right|_{2}$ is greater than or equal to the $i$ th minimum and, since the basis is reduced, the product of the norms of the vectors of the basis is of the same order as the determinant of $\Lambda^{j+1}$ and, thus, as the product of the minima. It follows that, for each $i,\left|e_{i}\right|_{2}$ is of the same order as the $i$ th minimum.

Finally, if $r$ is in $\left[(\sqrt{3} / 2)^{k}\left|e_{k}\right|_{2} / 6, Q_{j}^{-w}\right]$, we deduce from (8) that

$$
\begin{aligned}
\frac{\mu(B(x, r))}{r^{s}} & \leq \frac{\mu_{j+1}\left(B\left(x, r+Q_{j+1}^{-w}\right)\right)}{r^{s}} \\
& \leq \frac{m_{j+1} \operatorname{card} \Lambda^{j+1} \cap B\left(x, r+Q_{j+1}^{-w}+\left|e_{1}\right|_{2}\right)}{r^{s}} \\
& \ll \frac{m_{j+1}}{r^{s}} r^{k} Q_{j+1} \ll m_{j} \frac{Q_{j}^{w k}}{Q_{j+1}} r^{k-s} Q_{j+1} \\
& \ll m_{j} Q_{j}^{w k}\left(Q_{j}^{-w}\right)^{k-s} \ll m_{j-1} Q_{j-1}^{w k} Q_{j}^{s w-1} .
\end{aligned}
$$

STEP 6. To conclude, it is sufficient to define inductively the sequence $\left(n_{j}\right)_{j \geq 1}$ such that:

- the uniform distribution condition stated in Step 2 holds;

- $\left(m_{j} Q_{j}^{w k} Q_{j+1}^{w s-1}\right)_{j}$ tends to zero as $j \rightarrow \infty$;

- $\left.\left(m_{j} Q_{j}^{w k} \cdot \text { (last minimum of } \Lambda^{j+1}\right)^{k-s}\right)_{j}$ tends to zero as $j \rightarrow \infty$.

This is possible since $w s-1<0$ and thanks to Lemma 9. Then the results obtained in Step 5 show that, for any $x$ in $\mathcal{K}$, we have

$$
\lim _{r \rightarrow 0} \frac{\mu(B(x, r))}{r^{s}}=0 .
$$

If we take $s$ arbitrarily close to $1 / w$, this proves that the Hausdorff dimension of $\mathcal{W}_{w}(\underline{\alpha})$ is at least $1 / w$. Theorem 3 now follows from Proposition 1.

6. Proof of Proposition 1 and Theorems 4 and 5. Theorems 4 and 5 are particular cases of a more general result on systems of linear forms. For $u>0$ and $A$ in $M_{n, m}(\mathbb{R})$, set

$$
\mathcal{U}_{u}(A)=\left\{\underline{\xi} \in \mathbb{R}^{n}:\|A \underline{x}-\underline{\xi}\| \leq 1 /|\underline{x}|^{u} \text { for infinitely many } \underline{x} \text { in } \mathbb{Z}^{m}\right\},
$$


where $|\underline{x}|$ denotes the maximum of the absolute values of the entries of the integer $m$-tuple $\underline{x}$, and $A \underline{x}$ is the (usual) product of the matrix $A$ by the column vector $\underline{x}$.

This section is devoted to the proof of the following assertion.

TheOREM 6. Let $n, m$ be positive integers and $u \geq m / n$ be a real number. Then for almost all $A$ in $M_{n, m}(\mathbb{R})$, we have

$$
\operatorname{dim} \mathcal{U}_{u}(A)=m / u \text {. }
$$

Theorems 4 and 5 follow straightforwardly from Theorem 6 . Actually, we prove a slightly stronger result, since (in contrast to $\mathcal{V}_{v}(\underline{\alpha})$ and $\mathcal{W}_{w}(\underline{\alpha})$ ) no positive constant $c$ is involved in the definition of $\mathcal{U}_{u}(A)$.

Let $A$ be in $M_{n, m}(\mathbb{R})$. The Hausdorff-Cantelli lemma easily yields the upper bound $\operatorname{dim} \mathcal{U}_{u}(A) \leq m / u$. Indeed, for any positive integer $n$, we set

$$
E_{n}=\bigcup_{\substack{|\underline{x}|=n \\ \underline{x} \in \mathbb{Z}^{m}}}\left\{\underline{\xi} \in \mathbb{R}^{n}:\|A \underline{x}-\underline{\xi}\| \leq 1 / n^{u}\right\} \cap[0,1]^{n}
$$

and $\varepsilon_{n}=n^{-u}$. Let $s>m / u$ be real. Observe that

$$
\mathcal{H}_{\varepsilon_{n}}^{s}\left(E_{n}\right) \ll n^{m-1} \varepsilon_{n}^{s} \ll n^{m-1-u s},
$$

where the constant implied in $\ll$ only depends on $n$ and $m$. Since $\mathcal{U}_{u}(A) \cap$ $[0,1]^{n}=\limsup _{n \rightarrow \infty} E_{n}$ and $m-1-u s<-1$, we infer from Lemma 7 that

$$
\mathcal{H}^{s}\left(\mathcal{U}_{u}(A) \cap[0,1]^{n}\right)=0 .
$$

Consequently, the Hausdorff dimension of $\mathcal{U}_{u}(A)$ is at most $m / u$. The same argument shows that, for any positive real $c$, the Hausdorff dimension of the set

$$
\left\{\underline{\xi} \in \mathbb{R}^{n}:\|A \underline{x}-\underline{\xi}\| \leq c /|\underline{x}|^{u} \text { for infinitely many } \underline{x} \text { in } \mathbb{Z}^{m}\right\}
$$

is at most $m / u$. This proves Proposition 1 .

However, the reverse inequality is slightly more difficult to obtain. Our proof uses on the one hand a classical result of Cassels [5], asserting that almost all matrices $A$ in $M_{n, m}(\mathbb{R})$ share a certain approximation property. On the other hand, we use the notion of ubiquitous systems, introduced by Dodson, Rynne and Vickers [8] to get the expected lower bound for the dimension of $\mathcal{U}_{u}(A)$.

First, we recall some results about Diophantine approximation.

Definition 2. A matrix $A$ in $M_{n, m}(\mathbb{R})$ is regular if there exists $\delta>0$ and infinitely many positive integers $X$ such that

$$
\inf \left\{\|A \underline{x}\|: \underline{x} \in \mathbb{Z}^{m}, \underline{x} \neq 0,|\underline{x}| \leq X\right\} \geq \delta X^{-m / n} .
$$

It follows from the Borel-Cantelli lemma that almost all matrices are regular (see e.g. [5, p. 92]). 
We further need a transference theorem between homogeneous and inhomogeneous approximation.

Theorem B. Let $A$ be in $M_{n, m}(\mathbb{R})$. Suppose that for every non-zero $\underline{x}$ in $\mathbb{Z}^{m}$ with $|\underline{x}| \leq X_{0}$ we have $\|A \underline{x}\|>c$. Then, for all $\underline{\xi}$ in $\mathbb{R}^{n}$, there exists $\underline{x}$ in $\mathbb{Z}^{m}$ such that

$$
\|A \underline{x}-\underline{\xi}\| \leq \frac{1}{2}\left(X_{0}^{-m} c^{-n}+1\right) c \quad \text { and } \quad|\underline{x}| \leq \frac{1}{2}\left(X_{0}^{-m} c^{-n}+1\right) X_{0} .
$$

Proof. This is [5, Theorem VI, p. 82].

We shall deduce from Theorem B that the lower bound $\operatorname{dim} \mathcal{U}_{u}(A) \geq m / u$ holds for every regular matrix $A$.

We first recall some facts about ubiquitous systems. Let $\Omega$ be a bounded open subset of $\mathbb{R}^{n},\left(S_{\alpha}\right)_{\alpha \in J}$ be a family of subsets of $\Omega, \mu: J \rightarrow \mathbb{R}^{+}$be a positive function, and $\psi: \mathbb{R}^{+} \rightarrow \mathbb{R}^{+}$be a non-increasing function tending to 0 as $x \rightarrow \infty$. Finally, set

$$
S_{\alpha}(\varrho)=\left\{\underline{\xi} \in \Omega: d\left(\underline{\xi}, S_{\alpha}\right)<\varrho\right\}
$$

and

$$
\mathcal{L}\left(S_{\alpha}, \mu, \psi\right)=\left\{\underline{\xi} \in \Omega: d\left(\underline{\xi}, S_{\alpha}\right) \leq \psi(\mu(\alpha)) \text { for infinitely many } \alpha \text { in } J\right\} .
$$

In what follows, we denote by $\operatorname{diam} \mathcal{C}$ the diameter of a hypercube $\mathcal{C}$, that is, the supremum of the distances between any two points of $\mathcal{C}$. Assume that the following hypothesis is satisfied. For each $j$, there exists a Lebesgue measurable subset $E(j)$ and a positive number $\lambda(j)$ such that

$$
\lim _{j \rightarrow \infty}|\Omega \backslash E(j)|=0, \quad \lim _{j \rightarrow \infty} \lambda(j)=0,
$$

and for any hypercube $\mathcal{C} \subset \Omega$ with $\operatorname{diam} \mathcal{C}=\lambda(j)$ and $\frac{1}{2} \mathcal{C} \cap E(j) \neq \emptyset$, there exist a real number $d$ and $\alpha$ in $J$, with $\mu(\alpha) \leq j$ and $0 \leq d \leq j$, such that for all $\varrho$ satisfying $0<\varrho \leq \lambda(j)$, we have

$$
\left|\mathcal{C} \cap S_{\alpha}(\varrho)\right| \gg \varrho^{n-d}(\operatorname{diam} \mathcal{C})^{d}
$$

and

$$
\left|\mathcal{C}^{\prime} \cap \mathcal{C} \cap S_{\alpha}(\varrho)\right| \ll \varrho^{n-d}\left(\operatorname{diam} \mathcal{C}^{\prime}\right)^{d},
$$

where $\mathcal{C}^{\prime}$ is any hypercube in $\Omega$ with $\operatorname{diam} \mathcal{C}^{\prime} \leq \lambda(j)$. The system $\left(S_{\alpha}, \mu\right)$ is called a ubiquitous system relative to $\lambda$. The real number $d$ is called the dimension of $\left(S_{\alpha}\right)$. The following result was proved by Dodson, Rynne and Vickers [8].

Theorem C. Suppose that $\left(S_{\alpha}, \mu\right)$ is a ubiquitous system with respect to $\lambda$ and that $\psi: \mathbb{R}^{+} \rightarrow \mathbb{R}^{+}$is a decreasing function. Let $t=d+\gamma(n-d)$, where

$$
\gamma=\min \left\{1, \limsup _{j \rightarrow \infty} \frac{\log \lambda(j)}{\log \psi(j)}\right\} \text {. }
$$


Then

$$
\operatorname{dim} \mathcal{L}\left(S_{\alpha}, \mu, \psi\right) \geq t
$$

Proof. This is Theorem 1 from [8].

We are now ready to prove Theorem 6 .

Proof of Theorem 6. Let $A$ be a regular matrix in $M_{n, m}(\mathbb{R})$. There exist $\delta>0$ and an increasing sequence of integers $\left(x_{p}\right)_{p \geq 0}$ with $x_{p} \rightarrow \infty$, such that for every $p$ we have

$$
\inf \left\{\|A \underline{x}\|: \underline{x} \in \mathbb{Z}^{m}, \underline{x} \neq 0,|\underline{x}| \leq x_{p}\right\} \geq \delta x_{p}^{-m / n} .
$$

By Theorem B, for any $\underline{\xi} \in \mathbb{R}^{n}$ there exists $\underline{x} \in \mathbb{Z}^{m}$ such that

$$
\begin{aligned}
\|A \underline{x}-\underline{\xi}\| & \leq \frac{1}{2}\left(x_{p}^{-m}\left(\delta x_{p}^{-m / n}\right)^{-n}+1\right) \delta x_{p}^{-m / n} \\
& =\frac{1}{2}\left(\delta^{-n}+1\right) \delta x_{p}^{-m / n}=\Delta \delta x_{p}^{-m / n}
\end{aligned}
$$

and

$$
|\underline{x}| \leq \frac{1}{2}\left(\delta^{-n}+1\right) x_{p}=\Delta x_{p},
$$

where $\Delta=\frac{1}{2}\left(\delta^{-n}+1\right)$. For a positive integer $p$, set $S_{p}=\{A \underline{x}:|\underline{x}| \leq$ $\left.\Delta x_{p}\right\}+\mathbb{Z}^{n}$, and $\mu(p)=p$. We claim that $\left(S_{p}, \mu\right)$ is a ubiquitous system with respect to $\lambda(p)=\Delta \delta x_{p}^{-m / n}$ (and $\left.\Omega=\right] 0,1\left[^{n}\right.$ ). Indeed, by (12) and (13), any hypercube $\mathcal{C}$ of diameter at least $\lambda(p)=\Delta \delta x_{p}^{-m / n}$ contains at least one point of $S_{p}$; therefore, we have

$$
\left|\mathcal{C} \cap\left\{\underline{\xi} \in \Omega: d\left(\underline{\xi}, S_{p}\right)<\varrho\right\}\right| \gg \varrho^{n}
$$

for any $\varrho$ satisfying $0<\varrho \leq \lambda_{p}$, and (10) holds with $d=0$. As for (11), consider the sets

$$
T_{p}(\underline{x})=A \underline{x}+\left\{A \underline{y}: \underline{y} \in \mathbb{Z}^{m},|\underline{y}| \leq \frac{1}{2} x_{p}\right\}+\mathbb{Z}^{n}, \quad \underline{x} \in \mathbb{Z}^{m} .
$$

There exists an integer $a$ depending only on $\delta$ and $n$ such that for all $p$ there exist $\underline{x}_{1}, \ldots, \underline{x}_{a} \in \mathbb{Z}^{m}$ with

$$
S_{p} \subset \bigcup_{i=1}^{a} T_{p}\left(\underline{x}_{i}\right) .
$$

Let $\mathcal{C}^{\prime}$ be a hypercube with diameter less than $\lambda(p)=\Delta \delta x_{p}^{-m / n}$. Since the distance between two points of $T_{p}(\underline{x})$ is at least $\delta x_{p}^{-m / n}$, the hypercube $\mathcal{C}^{\prime}$ contains at most $\Delta^{-n}$ points of $T_{p}(\underline{x})$. We get

$$
\left|\mathcal{C}^{\prime} \cap\left\{\underline{\xi} \in \Omega: d\left(\underline{\xi}, T_{p}(\underline{x})\right) \leq \varrho\right\}\right| \ll \varrho^{n}
$$

and therefore

$$
\left|\mathcal{C}^{\prime} \cap\left\{\underline{\xi} \in \Omega: d\left(\underline{\xi}, S_{p}\right)<\varrho\right\}\right| \ll \varrho^{n} .
$$


Consequently, (11) holds for $d=0$. Let $\psi: \mathbb{R}^{+} \rightarrow \mathbb{R}^{+}$be a non-increasing function such that $\psi(p)=x_{p}^{-u}$. Theorem $\mathrm{C}$ yields the lower bound

$$
\begin{aligned}
\operatorname{dim} \mathcal{L}\left(S_{\alpha}, \mu, \psi\right) & \geq n \min \left\{1, \limsup _{p \rightarrow \infty} \frac{\log \lambda(p)}{\log \psi(p)}\right\} \\
& =n \min \left\{1, \limsup _{p \rightarrow \infty} \frac{\log \Delta \delta x_{p}^{-m / n}}{\log x_{p}^{-u}}\right\}=\min \left\{n, \frac{m}{u}\right\} .
\end{aligned}
$$

Since we have

$$
\mathcal{L}\left(S_{p}, \mu, \psi\right) \subset \mathcal{U}_{u}(A) \cup\left(A \mathbb{Z}^{m}+\mathbb{Z}^{n}\right),
$$

the proof is finished.

\section{References}

[1] L. Babai, On Lovasz' lattice reduction and the nearest lattice point problem, Combinatorica 6 (1986), 1-13.

[2] V. I. Bernik and M. M. Dodson, Metric Diophantine Approximation on Manifolds, Cambridge Tracts in Math. 137, Cambridge Univ. Press, 1999.

[3] A. S. Besicovitch, Sets of fractional dimension (IV): on rational approximation to real numbers, J. London Math. Soc. 9 (1934), 126-131.

[4] Y. Bugeaud, A note on inhomogeneous Diophantine approximation, Glasgow Math. J. 45 (2003), 105-110.

[5] J. W. S. Cassels, An Introduction to Diophantine Approximation, Cambridge Tracts in Math. and Math. Phys. 99, Cambridge Univ. Press, 1957.

[6] N. Chevallier, Meilleures approximations d'un élément du tore $\mathbb{T}^{2}$ et géométrie de la suite des multiples de cet élément, Acta Arith. 78 (1996), 19-35.

[7] M. M. Dodson, A note on metric inhomogeneous Diophantine approximation, J. Austral. Math. Soc. Ser. A 62 (1997), 175-185.

[8] M. M. Dodson, B. P. Rynne, and J. A. G. Vickers, Diophantine approximation and a lower bound for Hausdorff dimension, Mathematika 37 (1990), 59-73.

[9] K. Falconer, Fractal Geometry: Mathematical Foundations and Applications, Wiley, 1990.

[10] V. Jarník, Diophantischen Approximationen und Hausdorffsches Maß, Mat. Sb. 36 (1929), 371-382.

[11] A. Ya. Khintchine, Über eine Klasse linearer diophantischer Approximationen, Rend. Circ. Mat. Palermo 50 (1926), 170-195.

[12] J. C. Lagarias, Some new results in simultaneous Diophantine approximation, in: Proc. Queen's Number Theory Conference 1979, P. Ribenboim (ed.), Queen's Paper in Pure Appl. Math. 54, Queen's Univ. Kingston, Ont., 1980, 453-474.

[13] J. Levesley, A general inhomogeneous Jarnik-Besicovitch theorem, J. Number Theory 71 (1998), 65-80.

[14] J. Schmeling and S. Troubetzkoy, Inhomogeneous Diophantine approximation and angular recurrence for polygonal billiards, Sb. Math. 194 (2003), 295-309.

[15] W. M. Schmidt, Badly approximable systems of linear forms, J. Number Theory 1 (1969), 139-154. 
[16] W. M. Schmidt, Diophantine Approximation, Lecture Notes in Math. 785, Springer, Berlin, 1980.

U.F.R. de mathématiques

Université Louis Pasteur

7, rue René Descartes

67084 Strasbourg, France

E-mail: bugeaud@math.u-strasbg.fr
Mathématiques Université de Haute Alsace 4 , rue des frères Lumière 68093 Mulhouse, France

E-mail: n.chevallier@univ-mulhouse.fr

Received on 22.11.2004

and in revised form on 6.2.2006 